\title{
Thermal Chains and Entrainment in Cumulus Updrafts. Part II: Analysis of Idealized Simulations
}

\author{
John M. Peters, ${ }^{a}$ Hugh Morrison, ${ }^{\mathrm{b}}$ Adam C. Varble,${ }^{\mathrm{c}}$ Walter M. Hannah, ${ }^{\mathrm{d}}$ \\ AND SCOTT E. GIANGRANDE ${ }^{\mathrm{e}}$ \\ ${ }^{\text {a }}$ Department of Meteorology, Naval Postgraduate School, Monterey, California; ${ }^{\mathrm{b}}$ National Center for \\ Atmospheric Research, Boulder, Colorado; ${ }^{\mathrm{c}}$ Pacific Northwest National Laboratory, Richland, Washington; \\ ${ }^{\mathrm{d}}$ Lawrence Livermore National Laboratory, Livermore, California; ${ }^{\mathrm{e}}$ Brookhaven National \\ Laboratory, Upton, New York
}

(Manuscript received 10 September 2019, in final form 5 July 2020)

\begin{abstract}
Research has suggested that the structure of deep convection often consists of a series of rising thermals, or "thermal chain," which contrasts with existing conceptual models that are used to construct cumulus parameterizations. Simplified theoretical expressions for updraft properties obtained in Part I of this study are used to develop a hypothesis explaining why this structure occurs. In this hypothesis, cumulus updraft structure is strongly influenced by organized entrainment below the updraft's vertical velocity maximum. In a dry environment, this enhanced entrainment can locally reduce condensation rates and increase evaporation, thus eroding buoyancy. For moderate-to-large initial cloud radius $R$, this breaks up the updraft into a succession of discrete pulses of rising motion (i.e., a thermal chain). For small $R$, this leads to the structure of a single, isolated rising thermal. In contrast, moist environments are hypothesized to favor plume-like updrafts for moderate-to-large $R$. In a series of axisymmetric numerical cloud simulations, $R$ and environmental relative humidity (RH) are systematically varied to test this hypothesis. Vertical profiles of fractional entrainment rate, passive tracer concentration, buoyancy, and vertical velocity from these runs agree well with vertical profiles calculated from the theoretical expressions in Part I. Analysis of the simulations supports the hypothesized dependency of updraft structure on $R$ and RH, that is, whether it consists of an isolated thermal, a thermal chain, or a plume, and the role of organized entrainment in driving this dependency. Additional three-dimensional (3D) turbulent cloud simulations are analyzed, and the behavior of these $3 \mathrm{D}$ runs is qualitatively consistent with the theoretical expressions and axisymmetric simulations.
\end{abstract}

KEYWORDS: Buoyancy; Convection; Entrainment; Updrafts/downdrafts; Cumulus clouds

\section{Introduction}

Over the past century, two primary conceptual models have served as a basis for understanding deep moist convection, and for representing moist convective processes in cumulus parameterizations (Emanuel 1994). The first conceptual model is the "steady-state plume" (e.g., Squires and Turner 1962), wherein deep convection is assumed to be a continuous column of rising fluid. Continuous entrainment along the edge of a steady-state plume is assumed to be driven by turbulence (hereafter "turbulent entrainment") that occurs on scales much smaller than the plume itself. This conceptual model originates from laboratory studies with a steady source of positive buoyancy at the lower boundary of a fluid. The steady-state plume model has gained traction in the atmospheric sciences community since it greatly reduces the complexity of theoretical equations that are used to understand deep convection. The other conceptual model for convection is the "bubble" or "thermal" model (e.g., Scorer and Ludlam 1953), wherein deep convection is assumed to be composed of discrete pulses of rising positive buoyancy anomalies that resemble

Corresponding author: J. Peters, jmpeters@nps.edu spherical vortices. In thermals, entrainment is primarily accomplished by organized flow structures that occur on the scale of the thermal itself (McKim et al. 2020), and laboratory studies have suggested that entrainment rates in thermals are larger than those in plumes by a factor of 2 (Morton et al. 1956; Scorer 1957). The thermal conceptual model also originates from laboratory studies, wherein thermals are formed when positive buoyancy is released intermittently, rather than continuously, at the lower fluid interface (e.g., Scorer 1957).

There has been a longstanding debate over whether the nature of moist convection in the atmosphere is plume-like or thermal-like (e.g., Yano 2014). Computational advances since the turn of the century have allowed for large-eddy simulations (LESs) of cumulus convection at unprecedented resolutions and domain sizes (e.g., Khairoutdinov et al. 2009; Sherwood et al. 2013; Romps and Charn 2015; Hernandez-Deckers and Sherwood 2016). These LESs, combined with high-resolution cloud radar and photogrammetric studies of cumulus convection (e.g., Damiani et al. 2006; Damiani and Vali 2007; Romps and Oktem 2015), have indicated the widespread occurrence of thermal-like structures within cumulus updrafts. This has arguably resolved part of the plume versus thermal debate, given evidence that thermals are nearly ubiquitous within 
a. $R=400, R H=42.5 \%$

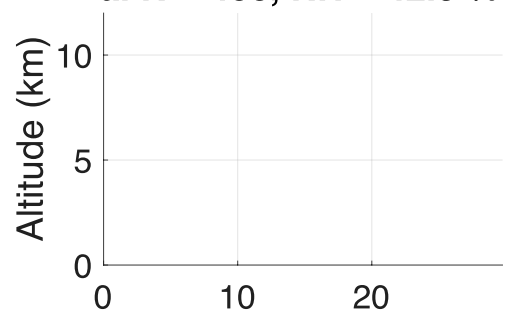

d. $R=1000, R H=42.5 \%$

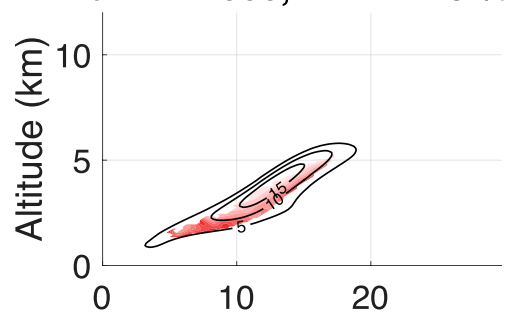

g. $R=1600, R H=42.5 \%$

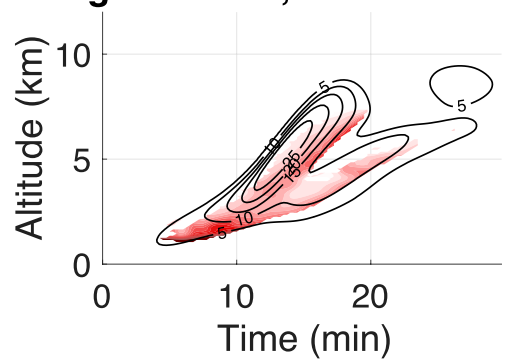

b. $R=600, \mathrm{RH}=42.5 \%$

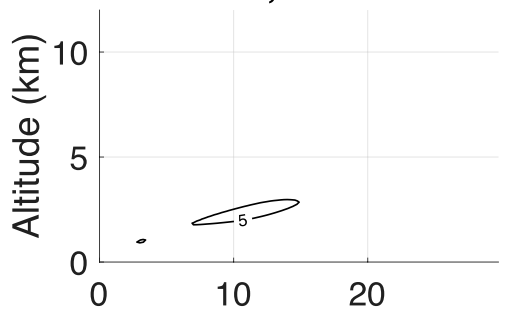

e. $R=1200, R H=42.5 \%$

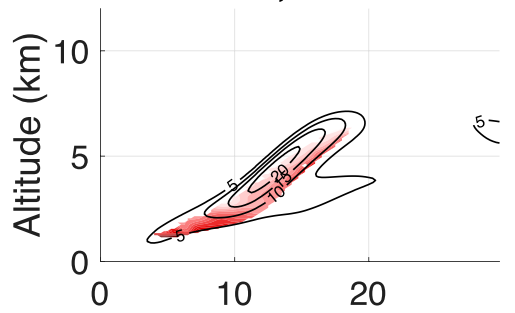

h. $R=1800, R H=42.5 \%$

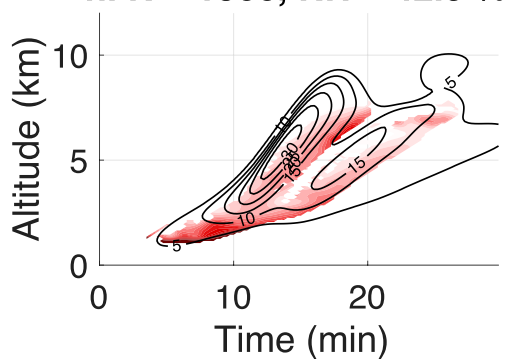

c. $R=800, R H=42.5 \%$

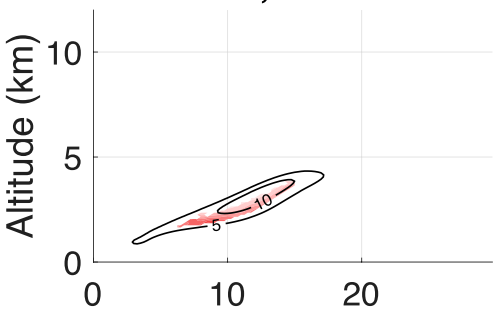

f. $R=1400, R H=42.5 \%$

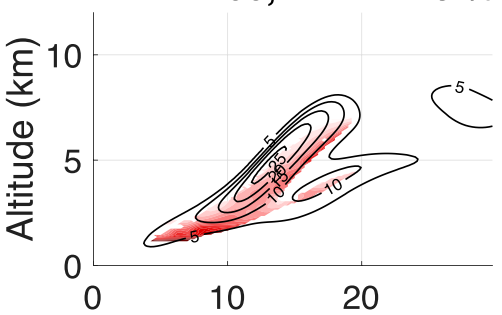

i. $\mathrm{R}=\mathbf{2 0 0 0}, \mathrm{RH}=\mathbf{4 2 . 5} \%$

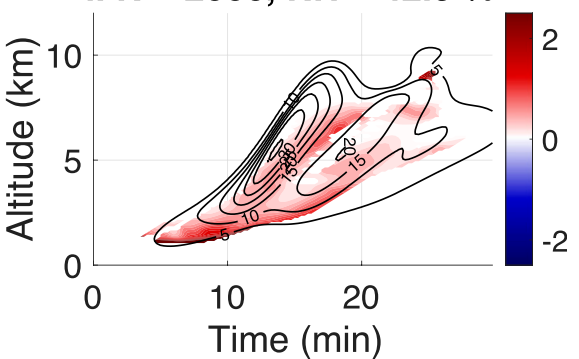

FIG. 1. Time vs height diagrams from the axisymmetric runs with $\mathrm{RH}=42.5 \%$ of fractional entrainment rate $\varepsilon\left(\right.$ shading, $\left.\mathrm{km}^{-1}\right)$ and vertical velocity (black contours at intervals of $5 \mathrm{~m} \mathrm{~s}^{-1}$, starting at $5 \mathrm{~m} \mathrm{~s}^{-1}$ ) at the updraft center. Initial bubble radii are (a) $400 \mathrm{~m}$ through (i) $2000 \mathrm{~m}$ at an interval of $200 \mathrm{~m}$.

moist convection. In many cases, these individual thermals, each with distinctive toroidal circulations and local vertical velocity maxima near their centers, rise in succession within clouds (e.g., Raymond and Blyth 1989; Blyth and Latham 1993; Damiani et al. 2006; Moser and Lasher-Trapp 2017; Peters et al. 2019). Individual cumulonimbus clouds may sometimes be composed of several rising thermals at a given time (e.g., Fig. 4 in Hernandez-Deckers and Sherwood 2016). We call this seemingly prevalent structure of cumulus convection a "thermal chain" (Morrison et al. 2020, hereafter Part I). Thermal chains are evident in LESs of weakly sheared midlatitude congestus (e.g., Moser and Lasher-Trapp 2017) and tropical deep convection (e.g., Romps and Oktem 2015; Hernandez-Deckers and Sherwood 2016), and comparatively strongly sheared midlatitude deep convection (e.g., Bryan and Fritsch 2002; Lebo and Morrison 2015).

What remains unclear is why thermal chains are a common mode of cumulus convection. What mechanisms determine whether updrafts have this structure, as opposed to that of a plume or isolated rising thermal? Resolving this question is scientifically important from the standpoint of improving our basic understanding of convective dynamics. It is also relevant to cumulus parameterizations, which are generally based on the framework of steady-state plumes (De Rooy et al. 2013, and references therein). Updraft structure has a strong influence on many aspects of convection relevant to parameterizations, entrainment in particular, and one of the primary uncertainties in cumulus parameterizations lies in their simplified treatments of entrainment (e.g., Zhou et al. 2012). Recent studies have hypothesized that traditional assumptions about entrainment related to the plume framework for convection in parameterizations have contributed to this uncertainty (e.g., Romps 2010; Hannah 2017). Improvements in understanding of the dynamics of moist thermals therefore directly relate to and potentially set the foundation for future improvements in cumulus parameterizations.

In Part I of this study, we developed theoretical expressions for cumulus updraft dynamics by simplifying the governing equations of mass continuity, vertical momentum, and cloud thermodynamics. Expressions for a passive tracer, buoyancy, and vertical velocity at various heights within updrafts were obtained, and they provide a simple quantitative model for the behavior of cumulus convection. From the results in Part I, we hypothesize that ascending buoyant updrafts rapidly develop a toroidal circulation (see Fig. 2 in 

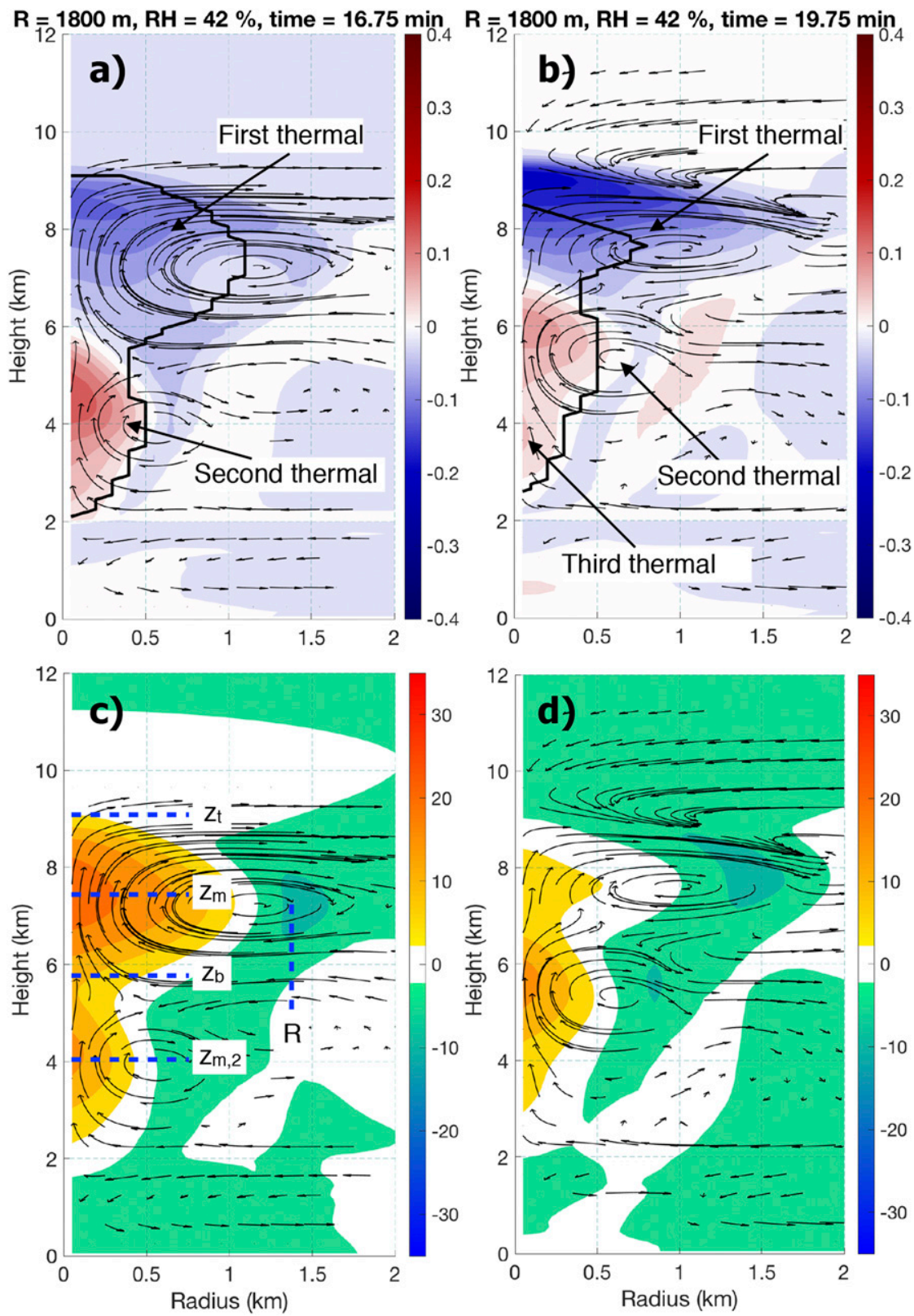

FIG. 2. Vertical cross sections of snapshots at (a),(c) 16.75 and (b),(d) $19.75 \mathrm{~min}$ from the axisymmetric simulation with initial bubble radius of $1800 \mathrm{~m}$ with $\mathrm{RH}=42.5 \%$. (a),(b) Buoyancy (shading, $\mathrm{m} \mathrm{s}^{-2}$ ), flow vectors (black arrows), and the cloud boundary (per the definition in the text, black solid line). (c),(d) Vertical velocity (shading, $\mathrm{m} \mathrm{s}^{-1}$ ) and flow vectors (black arrows).

Part I), which we will refer to as the "primary thermal." The inward branch of this circulation (i.e., flow toward the updraft center) locally enhances entrainment of environmental air. In dry environments, this leads to a local reduction of buoyancy relative to regions where dynamic entrainment is not locally enhanced. This buoyancy structure in turn modifies the flow and leads to a breakdown of updrafts into successive rising thermals—-the thermal chain structure. An essential part of this process is the entrainment of dry environmental air and the subsequent reduction of condensation rates and/or increase in evaporation, which greatly enhances the local reduction of buoyancy compared to surrounding levels. From this idea, we further hypothesize that thermal chains are most prevalent as the middle troposphere becomes drier, all else being equal, whereas convective structures are more plume-like in comparatively 
a. $R=400, R H=85 \%$
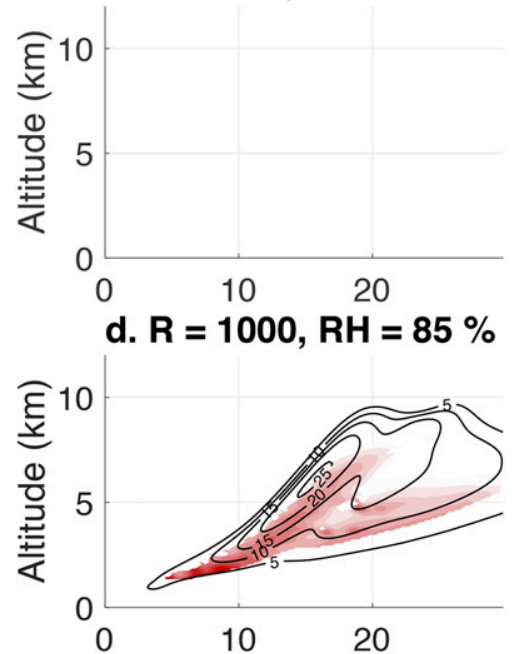

g. $R=1600$, $R H=85 \%$

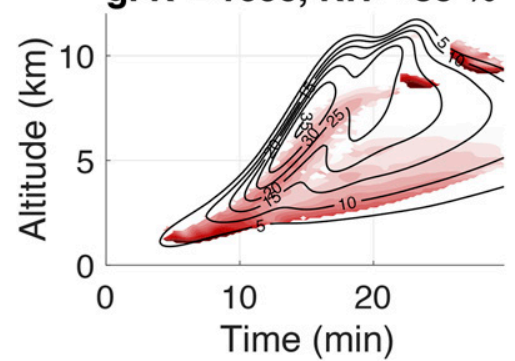

b. $\mathbf{R}=600, \mathbf{R H}=85 \%$
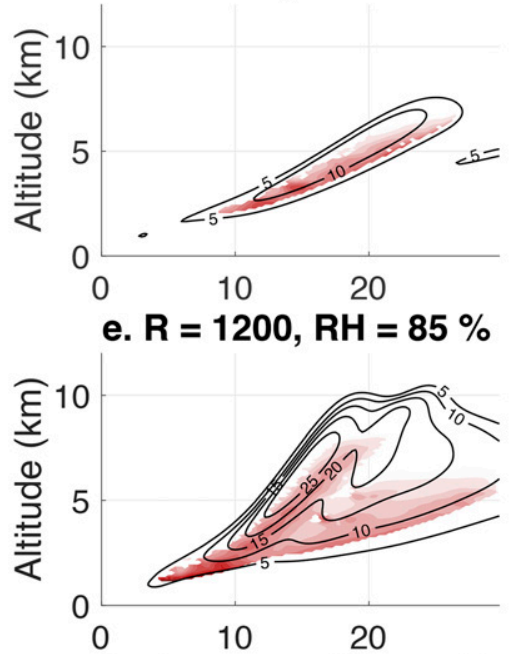

h. $R=1800, R H=85 \%$

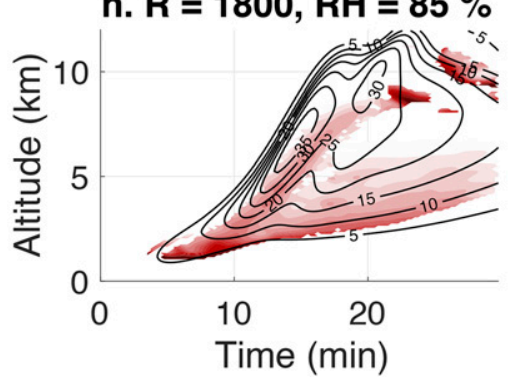

C. $\mathbf{R}=800, \mathbf{R H}=85 \%$

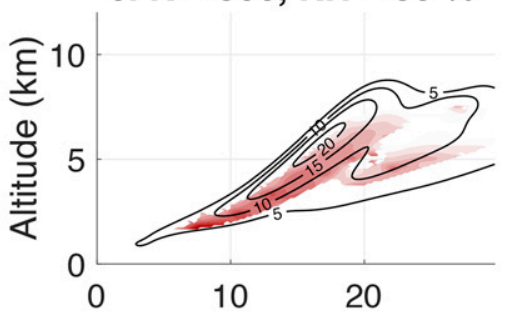

f. $R=1400, R H=85 \%$

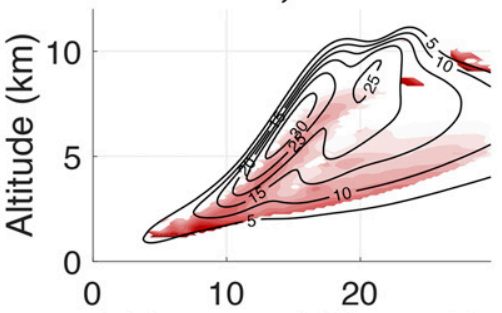

i. $R=2000, R H=85 \%$

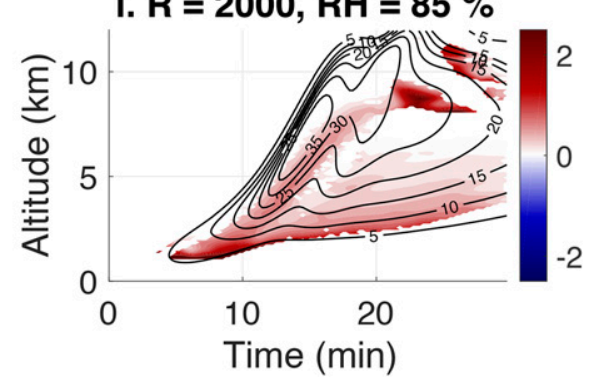

FIG. 3. As in Fig. 1, but for the $\mathrm{RH}=85 \%$ runs.

moist middle-tropospheric environments. ${ }^{1}$ Additionally, because of the dependence of entrainment-driven dilution on cloud radius, narrower clouds are hypothesized to be more thermal-like whereas wider clouds are more plumelike. For narrow clouds in a dry environment, strong dilution of core buoyancy from locally enhanced entrainment leads to a "pinching off" of updrafts from below, producing a single isolated rising thermal.

The purpose of this article is to test the aforementioned ideas using fully dynamical numerical simulations of moist deep convection. We ran a series of highly idealized axisymmetric simulations with simplified physics and dynamics over a range of middle-tropospheric environmental relative humidities, and with a range of cloud widths. The details of these simulations are described in section 2, and the results are presented in section 3 and directly compared to the theoretical expressions from Part I. We additionally performed comparatively sophisticated three-dimensional (3D) turbulent updraft

\footnotetext{
${ }^{1}$ In moist environments, more specifically we hypothesize that the plume-like structure of an ascending updraft resembles a "starting plume" (Turner 1962). In this structure, the rising plume head contains a toroidal circulation - the ascending primary thermal-with the flow behind the primary thermal resembling a steady-state plume.
}

simulations (also described in section 2) for selected environments to affirm the realism of these results, described in section 4. Finally, section 4 gives a summary and outlines general conclusions.

\section{Experiment design}

Cloud Model 1 (CM1; Bryan and Fritsch 2002) version 18 was used to run simulations that address the hypothesis outlined in the introduction. CM1 is a nonhydrostatic numerical model commonly used in idealized cloud studies. We configured the model with a compressible equation set using acoustic time splitting (e.g., Klemp and Wilhelmson 1978). Lower and upper boundaries were free-slip, and the horizontal and vertical grid spacing was $100 \mathrm{~m}$. Convection in all experiments was initiated using the method of Hannah (2017) by including a horizontally centered $1 \mathrm{~K}$ Gaussian warm and moist bubble in the initial conditions at a height of $500 \mathrm{~m}$. The initial relative humidity at a given level was held constant in the initial conditions. Microphysics was represented using the scheme of Morrison et al. (2005). Like in Morrison (2017) and Morrison and Peters (2018), the microphysics scheme was simplified to include only cloud water condensation and evaporation. Thus, we neglect complications arising from ice microphysics and precipitation. For simplicity and to allow a more direct comparison with the theoretical expressions from Part I, the effects of condensate loading on cloud buoyancy were also neglected. 

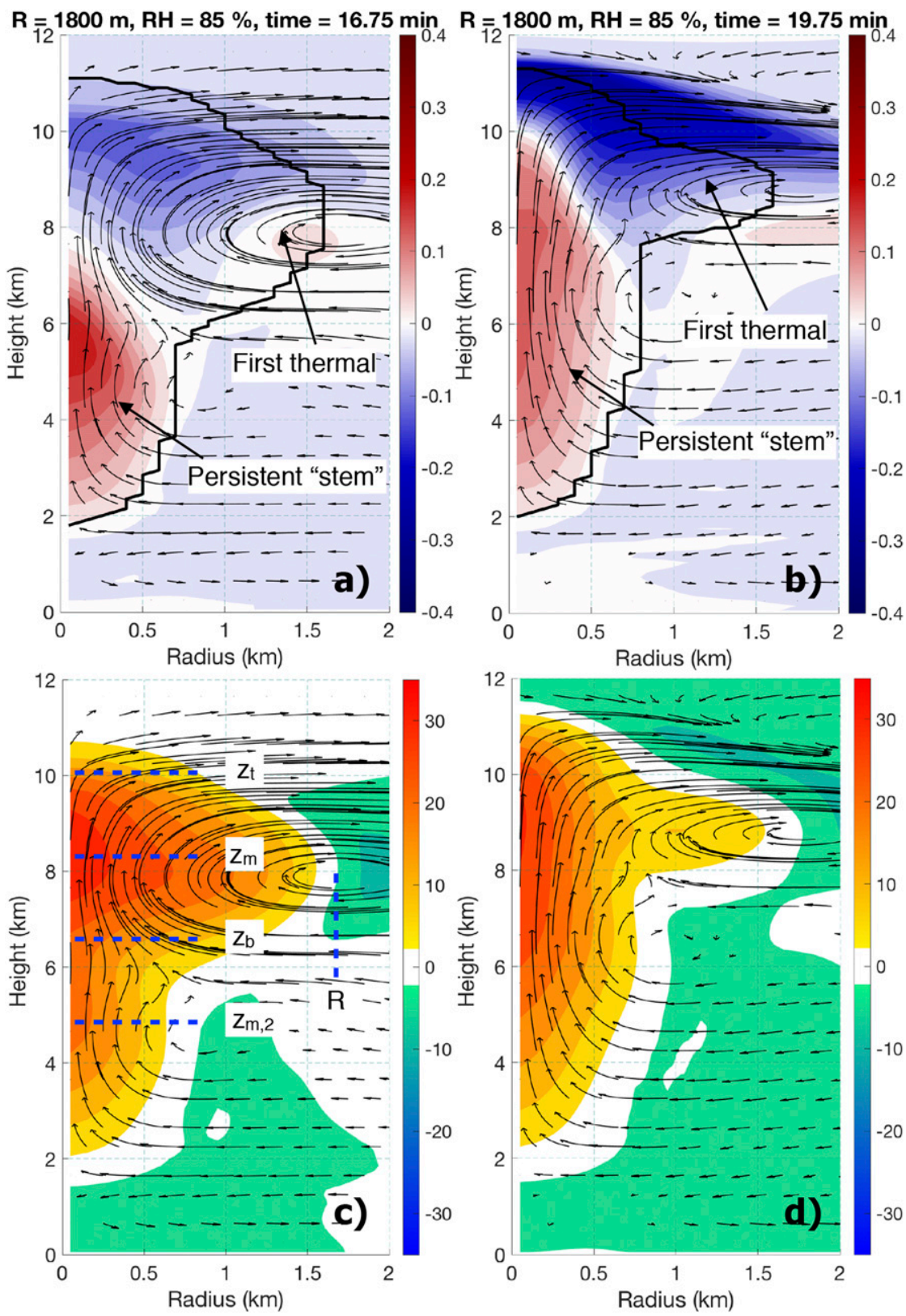

FIG. 4. As in Fig. 2, for the $1800 \mathrm{~m}$ run with $\mathrm{RH}=85 \%$.

Simulations used the analytic sounding of Weisman and Klemp (1982) with the boundary layer mixing ratio set to $14 \mathrm{~g} \mathrm{~kg}^{-1}$, and the relative humidity above $1.5 \mathrm{~km}$ set to $42.5 \%$ in the dry runs and $85 \%$ in the moist runs. The initial wind was set to zero everywhere. Specific details of the axisymmetric and 3D turbulent runs are described in the following subsections. Simulations were run for $30 \mathrm{~min}$, which was sufficient time for updrafts to rise through the troposphere and reach their termination heights.

The purpose of the axisymmetric runs was to compare numerical simulation results with the theoretical expressions from Part I. This simple axisymmetric framework allows a direct comparison with the theoretical expressions. We qualitatively compare overall updraft structure and behavior, and quantitatively compare vertical profiles of fractional entrainment rate, passive tracer, buoyancy, and vertical velocity at the center of the simulated updrafts with the analogous quantities calculated from the theoretical expressions. To facilitate this comparison, certain aspects of the updraft geometry and physics were simplified in the numerical model. These runs used the built-in axisymmetric mode option in CM1 with 500 radial points and 264 vertical points, with a vertical and radial grid spacing of $100 \mathrm{~m}$. The Smagorinsky-like subgrid-scale turbulence scheme was also modified to remove parameterized 

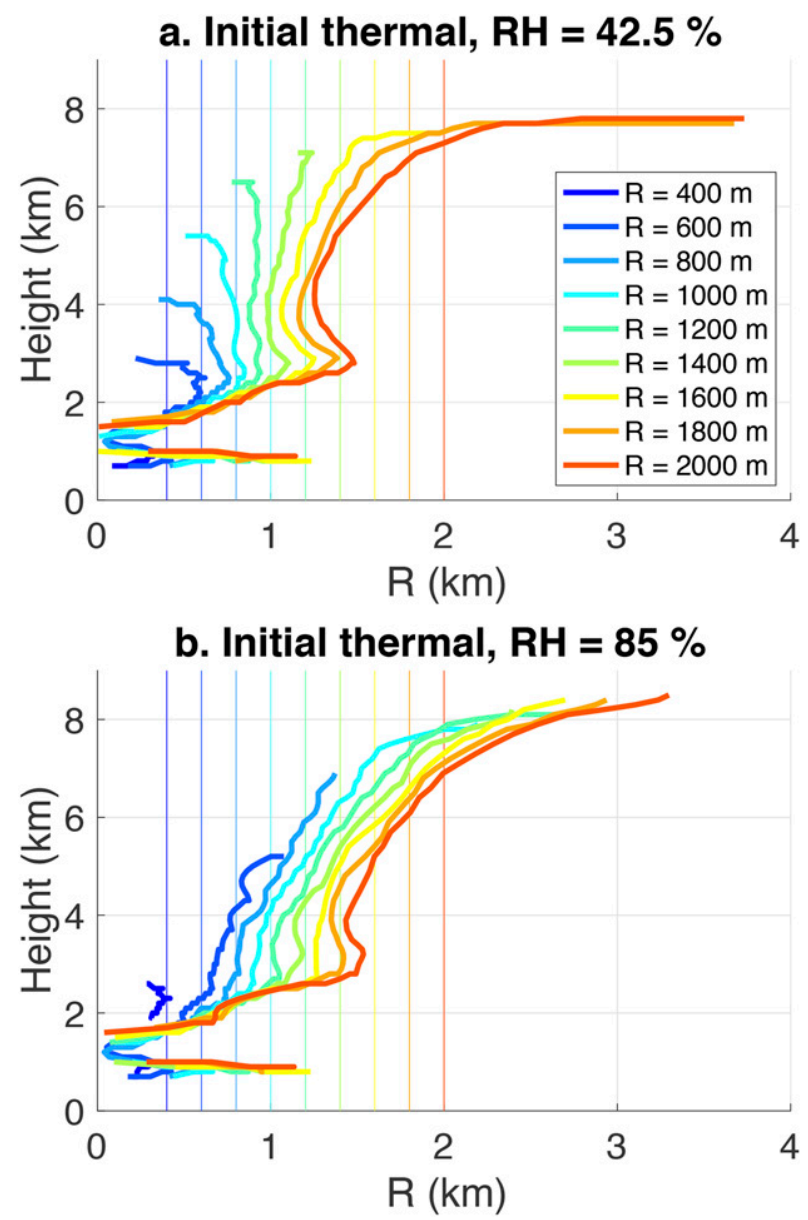

FIG. 5. Radius of tracked thermals ( $x$ axis, $\mathrm{km})$ as a function of thermal height ( $y$ axis, $\mathrm{km}$ ). The color of lines corresponds to the radius of the initial bubble [see the legend in (a)]. Plots show the primary thermal in the (a) $\mathrm{RH}=42.5 \%$ and (b) $\mathrm{RH}=85 \%$ runs.

subgrid-scale vertical mixing (note there is still implicit vertical mixing from the advection scheme). The horizontal mixing length was set to $500 \mathrm{~m}$ to account for the lack of realistic turbulence. This value is larger than the typical mixing length in the Smagorinsky scheme, which is typically set to the grid spacing. A similar approach was used in the numerical simulations described in Morrison (2017). Axisymmetric simulations were run with initial bubble radii of 400, 600, $800,1000,1200,1400,1600,1800$, and $2000 \mathrm{~m}$. Our range of initial radii is intended to encompass the thermal size range that previous studies have shown accomplishes the largest percentage of vertical mass flux in deep convection (i.e., 500-1500 m; Hernandez-Deckers and Sherwood 2016; Peters et al. 2019).

To bridge the complexity gap between the highly idealized nonturbulent axisymmetric simulations and realistic convection, which is turbulent, we also made qualitative comparisons of the theoretical model and axisymmetric simulations with 3D turbulent simulations. The fully 3D runs used a similar model configuration to Peters et al. (2019). The domain was configured as a cube with 264 points and a grid spacing of $100 \mathrm{~m}$ in all three directions. Random temperature perturbations with a maximum amplitude of $1 \mathrm{~K}$ were included below $2 \mathrm{~km}$ in the initial conditions to foster the development of turbulence. We used the Smagorinsky subgrid-scale turbulence scheme as included in the original CM1 source code (i.e., without the modifications described for the axisymmetric simulations). It was shown in Peters et al. (2019, Fig. 3a therein) that simulations with this setup produce realistic kinetic energy spectra (i.e., $-5 / 3$ slope over an inertial subrange) within $\sim 5 \mathrm{~min}$ of model initialization. Simulations were run with initial bubble radii of $500,1000,1500$, and $2000 \mathrm{~m}$. We ran four ensemble members for each configuration (combination of $R$ and $\mathrm{RH}$ ) of the 3D model. Different members had different random number seeds for generating the initial random temperature perturbations. Although there were differences among ensemble members, overall results were similar. Therefore, we only present results herein from a single member for each configuration.

\section{Analysis methods}

To make comparisons between simulated fractional entrainment rates and the theoretical entrainment estimates from Part I, we used the direct method for calculating entrainment described by Romps (2010). On a given vertical level, the local

TABLE 1. Bubble radius $R(\mathrm{~m})$ used to initialize simulations (first column) and the respective $R(\mathrm{~m})$ used in the theoretical expressions (second and third columns) based on the sizes of tracked simulated thermals. The $z_{t}$ values $(\mathrm{m})$ from Figs. 6-11 are shown in the fourth column and those from Figs. 12 and 13 are shown in the fifth column.

\begin{tabular}{|c|c|c|c|c|}
\hline $\begin{array}{l}\text { Initial bubble } R \text { in } \\
\text { simulations }\end{array}$ & $\begin{array}{c}\mathrm{RH}=42.5 \% R \text { in } \\
\text { expressions }\end{array}$ & $\begin{array}{c}\mathrm{RH}=85 \% R \text { in } \\
\text { expressions }\end{array}$ & $\begin{array}{l}z_{t} \text { for } B, C, \\
\quad \text { and } \varepsilon\end{array}$ & $z_{t}$ for $w$ \\
\hline 400 & 400 & 400 & 2500 & 2500 \\
\hline 600 & 700 & 800 & 3000 & 3000 \\
\hline 800 & 800 & 1100 & 4000 & 4000 \\
\hline 1000 & 1000 & 1200 & 4000 & 5000 \\
\hline 1200 & 1100 & 1300 & 5000 & 6000 \\
\hline 1400 & 1200 & 1400 & 6000 & 7000 \\
\hline 1600 & 1300 & 1500 & 6000 & 7000 \\
\hline 1800 & 1400 & 1600 & 6000 & 8000 \\
\hline 2000 & 1500 & 1700 & 6000 & 8000 \\
\hline
\end{tabular}


a. $\mathrm{R}=400, \mathrm{RH}=42.5 \%$
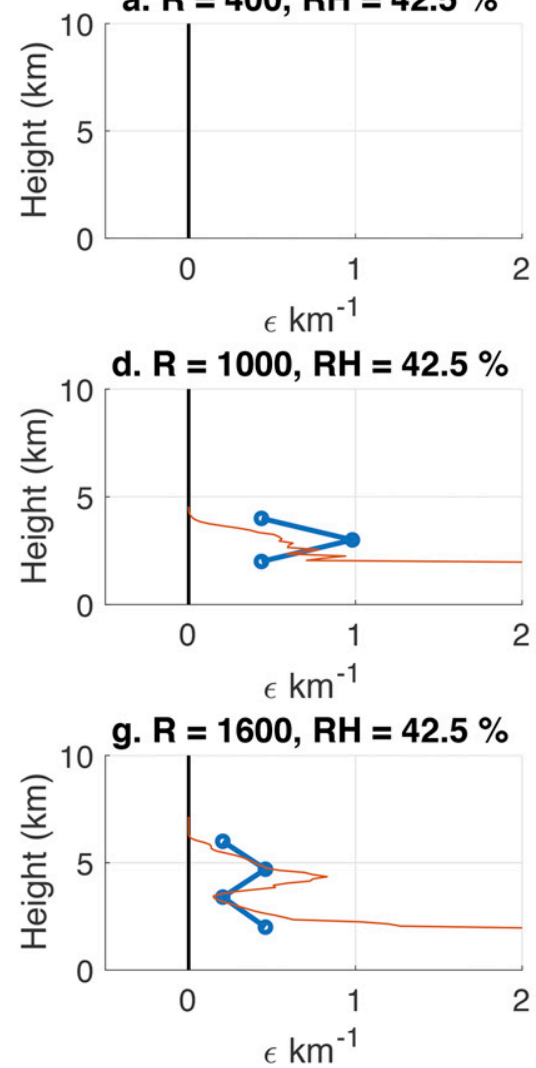

b. $\mathrm{R}=600, \mathrm{RH}=42.5 \%$
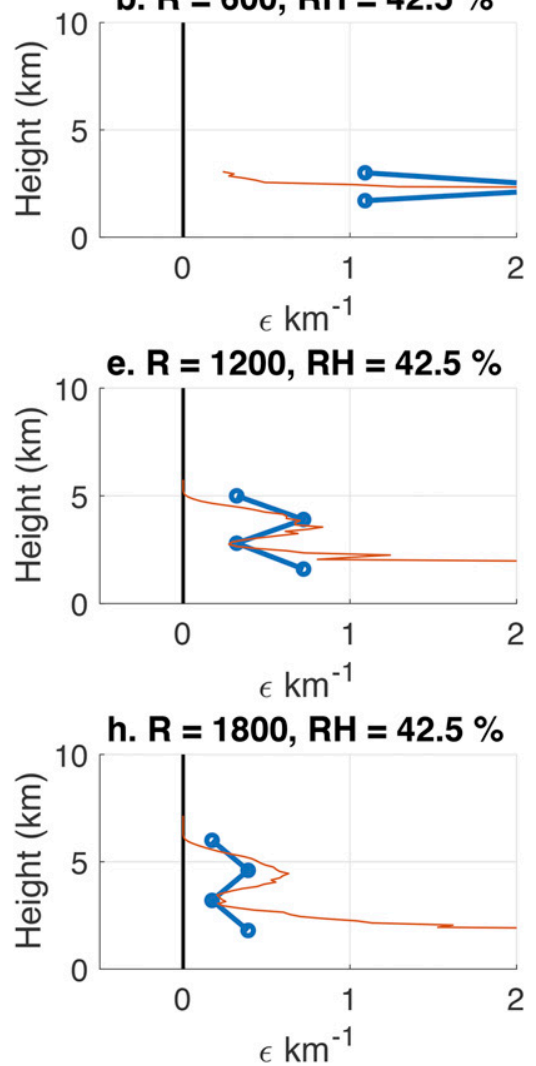

c. $\mathrm{R}=800, \mathrm{RH}=42.5 \%$
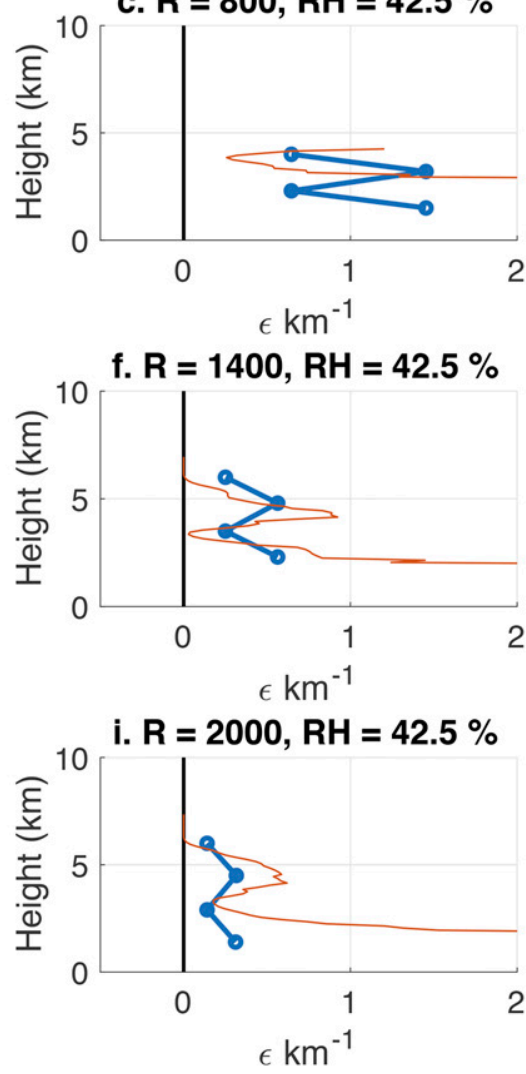

FIG. 6. Vertical profiles of fractional entrainment rate $\varepsilon$ from the $\mathrm{RH}=42.5 \%$ runs (red lines) and $\varepsilon$ calculated from the theoretical expressions (blue dots and lines) using the initial environment of the $\mathrm{RH}=42.5 \%$ runs. The blue dots indicate the heights at which the theoretical expressions were evaluated, and the blue lines connecting these dots are added simply for illustration.

entrainment $(e)$ and detrainment $(d)$ rates (with units of $\mathrm{kg} \mathrm{s}^{-1} \mathrm{~m}^{-3}$ ) at a given grid point are defined as

$$
e \equiv \max \left[\frac{d}{d t}(\rho \kappa), 0\right], \quad d \equiv \max \left[-\frac{d}{d t}(\rho \kappa), 0\right],
$$

where $\kappa=1$ defines the cloud area and $\kappa=0$ elsewhere. Here, the updraft volume is defined as having $w>3 \mathrm{~m} \mathrm{~s}^{-1}$ and cloud water mixing ratio $q_{c}>10^{-5} \mathrm{~kg} \mathrm{~kg}^{-1}$. Details of the numerics involved in these computations can be found in Romps (2010). From Eq. (1), we define fractional entrainment $\left(\varepsilon_{\text {sim }}\right)$ and detrainment $\left(\delta_{\text {sim }}\right)$ length scales as

$$
\varepsilon_{\mathrm{sim}} \equiv \frac{\iint(\varepsilon) d A}{\iint(\rho w \kappa) d A}, \quad \delta_{\mathrm{sim}} \equiv \frac{\iint(\delta) d A}{\iint(\rho w \kappa) d A},
$$

where $\iint(\cdots) d A$ is the horizontal integral over the model domain.

Note that as discussed in Part I, the comparison between the direct calculation of $\varepsilon$ in Eq. (1), and the theoretical $\varepsilon$ from Part I, is not necessarily "apples to apples." Whereas the direct calculation measures the flux of mass across the thermal boundary, the theoretical formula represents the turbulent entrainment rate that must occur to give the cloud core its tracer concentration $C$ at a given height. In fact, the direct calculation explicitly neglects turbulent mixing once entrained air has passed into the cloud, whereas the theoretical formula explicitly represents this "internal" turbulent mixing. Finally, the theoretical formula assumes that all entrained air has properties of the far-field background environment, whereas the direct calculation allows for horizontal heterogeneity in the properties of entrained air (i.e., clouds may reentrain air that has previously been detrained). These conceptual differences lead to some intrinsic quantitative differences between the direct calculation and theory. However, as will be shown later, the theoretical formula qualitatively embodies the behavior of directly calculated entrainment in the simulations despite these caveats.

Finally, it was necessary to determine the sizes of simulated thermals within the axisymmetric simulations in order to compare simulations with the theoretical expressions. To estimate thermal sizes, we used a simplified version of the tracking procedure described by Peters et al. (2019), which itself is a modified version of the tracking procedure introduced by Hernandez-Deckers and Sherwood (2016). In this method, model data were output every $15 \mathrm{~s}$. At each output time, we found local maxima in vertical velocity that exceeded $3 \mathrm{~m} \mathrm{~s}^{-1}$. 

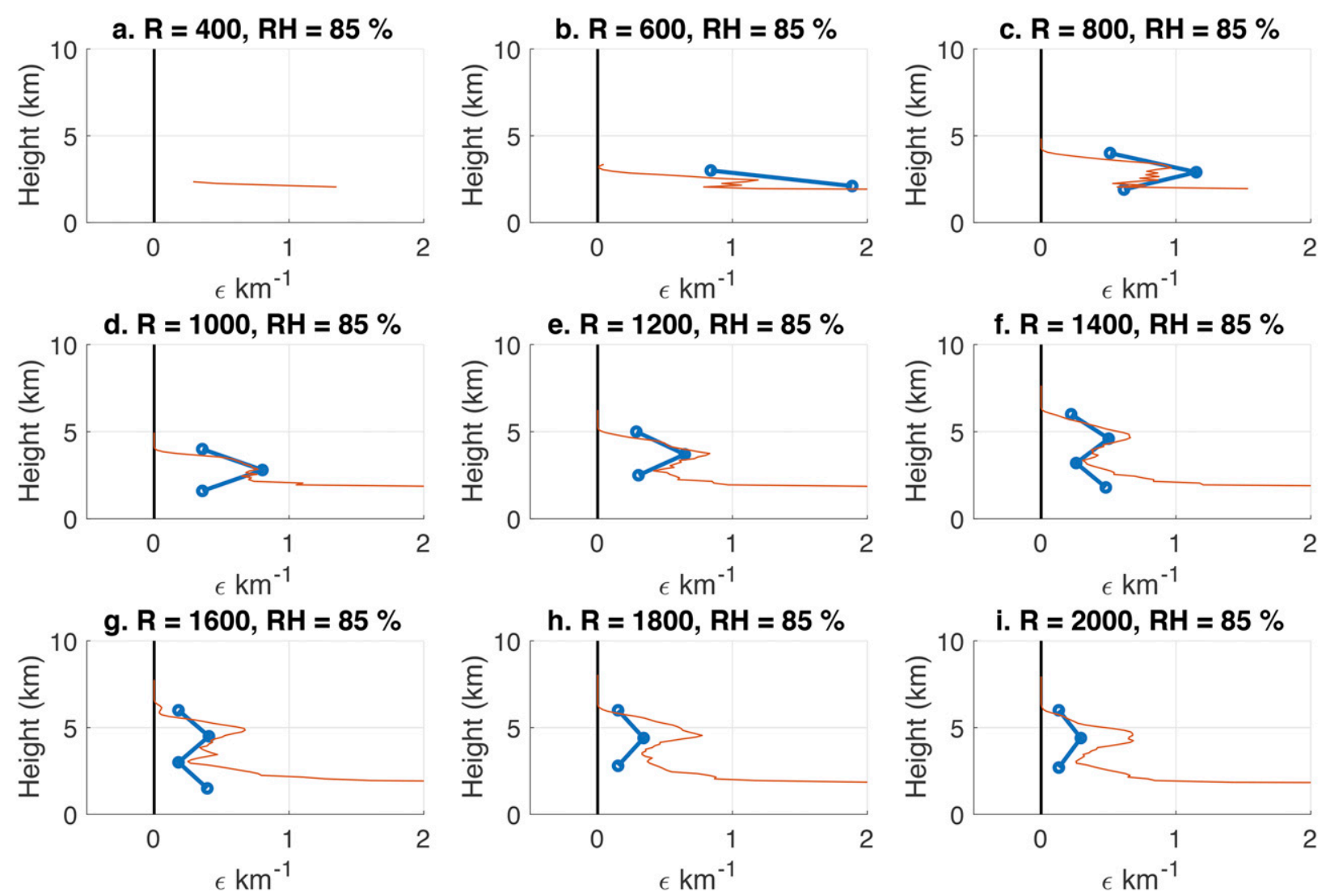

FIG. 7. As in Fig. 6, but for the RH $=85 \%$ runs.

The center point of the primary thermal was considered to be the highest maxima in vertical velocity $(w)$ for simulation times where the circulation structure of the primary thermal was still evident in model output. The ascent rate of the primary thermal was then assumed to match with the ascent rate of the topmost maximum in $w$. To determine thermal size, a sphere with increasing radius was centered at the topmost point of maximum $w$ until the volume averaged $w$ matched the thermal ascent rate. This sphere was used as an estimate for the thermal's radius.

\section{Results}

We first provide a brief overview of the characteristics of the axisymmetric simulations in this section, and qualitatively evaluate the consistency of the behavior of simulated thermals with the theoretical expressions. This is followed by a direct quantitative comparison between quantities from the simulated updrafts and the profiles of quantities that are calculated from the theoretical expressions.

\section{a. Characteristics of simulated thermals}

All simulations aside from those with an initial bubble with a radius of $400 \mathrm{~m}$ produced at least brief clouds with $w>5 \mathrm{~m} \mathrm{~s}^{-1}$ (Figs. 1 and 3). The dependency of simulated cloud behavior on the initial bubble radius and environmental RH was qualitatively consistent with the theorized dependencies in Part I.
For instance, the smallest bubbles in the low-RH runs (bubble radius of 400-1000 $\mathrm{m}$ ) either produced no updraft greater than $5 \mathrm{~m} \mathrm{~s}^{-1}$ (Fig. 1a), or a single isolated rising thermal (Figs. 1b-d). As initial bubble sizes became larger in the low-RH runs, primary and secondary thermals were produced, evident as distinct pairs of local $w$ maxima ascending with time in Figs. 1e-h. Both the primary and secondary thermals developed toroidal circulations that rose along with the thermals' maxima in $w$ (Figs. 2a-d). In the simulation with largest bubble size (2000 m) in the dry environment, a weak third maxima in $w$ was evident late in the simulation, indicating the development of a third thermal (Fig. 1i).

All of the high-RH runs (Figs. 3b-i), aside from the run with the $400 \mathrm{~m}$ initial bubble (Fig. 3a), produced a distinct primary thermal with a well-developed toroidal circulation (Figs. 4a,c). The runs with an initial bubble radius $>800 \mathrm{~m}$ in the high- $\mathrm{RH}$ runs produced updrafts that resembled starting plumes over the first 15-20 min. After this time, these runs produced secondary maxima in buoyancy and $w$; however, the character of these secondary maxima was distinctly different than that of low-RH runs. The secondary maxima appeared only after the first maxima had diminished; thus, the $w$ profiles generally only had a single $w$ maximum at any given time, in contrast to the low-RH runs. Moreover, the cloud region associated with the secondary $w$ maximum developed into a deep and persistent region of high $w$. The velocity structure of this secondary 

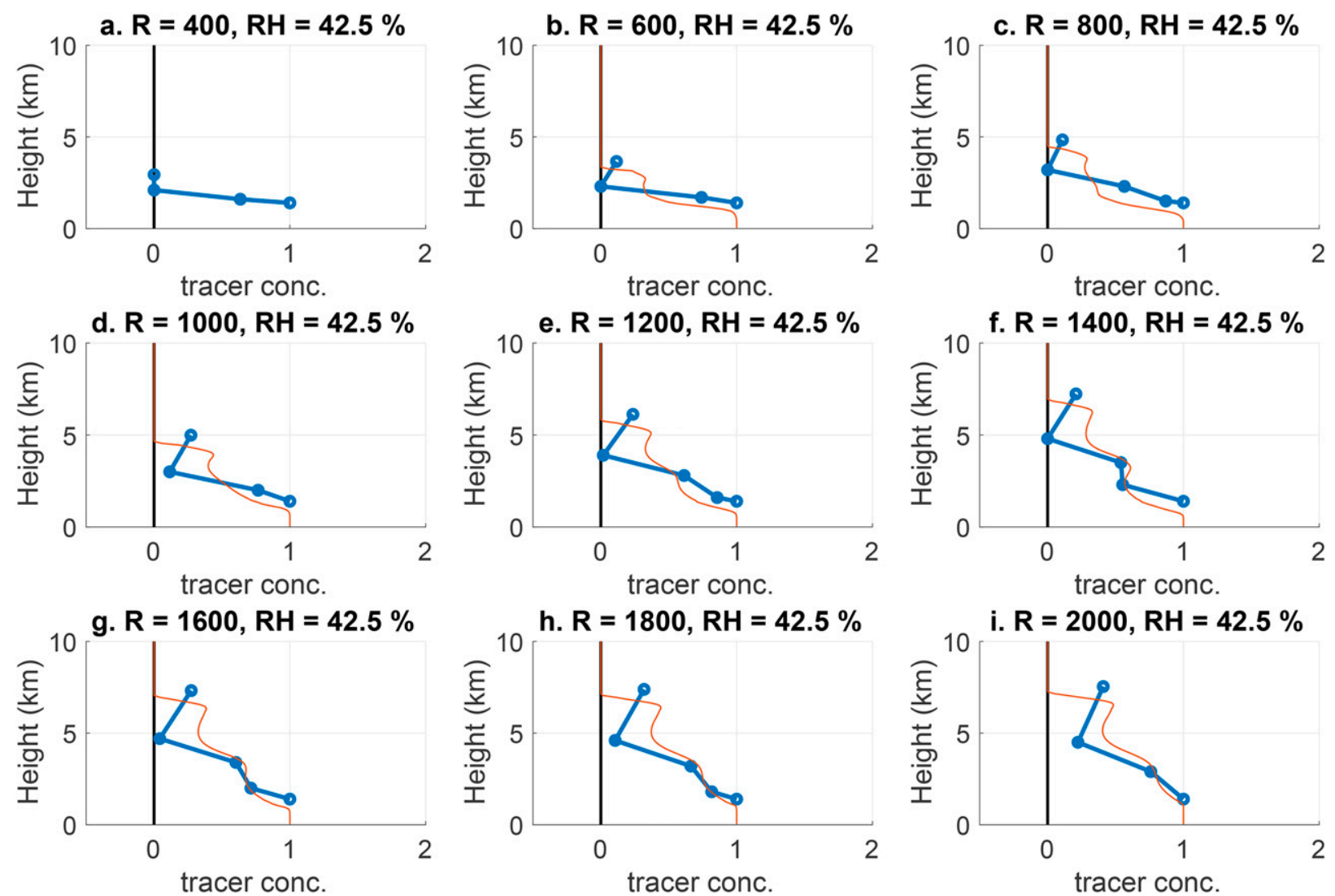

FIG. 8. As in Fig. 6, but for the passive tracer concentration at $r=0$ from the RH $=42.5 \%$ runs.

maximum somewhat resembled a thermal in the runs with smaller initial bubble radii (e.g., Figs. 3c,d), but became distinctly plume-like for the larger initial bubble radii with a lessdefined toroidal circulation and persistent deep region of rising motion (Figs. 4b,d).

Patterns of $\varepsilon$ among the runs were also consistent with the theoretical model. Local maxima in $\varepsilon$ occurred below the centers of rising maxima in $w$ (e.g., Figs. 1 and 3), and at the base of the plume-like structures that developed later in the high-RH runs (e.g., Figs. 3d-i). Local maxima in $\delta$ also occurred above rising maxima in $w$, and near the termination heights of thermals (not shown).

To determine how the radii of simulated thermals related to the initial bubble radius, we examine vertical profiles of the radii of tracked primary thermals (Fig. 5). The fluctuations in thermal size below $2 \mathrm{~km}$ are ignored as these variations are likely numerical artifacts of the tracking procedure. In the dry environment the radii were nearly constant with height until reaching $\sim 7 \mathrm{~km}$, above which they experienced a rapid increase in size (in the simulations with thermals attaining this height). In the moist environment the thermals' sizes increased somewhat as they ascended, with a relative increase from 2 to $6 \mathrm{~km}$ of about $20 \%-60 \%$. Above $\sim 7 \mathrm{~km}$ there was again a rapid increase in the size of thermals that reached this height. This rapid size increase near the end of thermal's lifetimes is related to deformation of thermal shapes as they reach their levels of neutral buoyancy and become negatively buoyant. Overall, thermal sizes were typically smaller than the initial bubble for large initial bubbles, and comparable to or larger than the initial bubble for small initial bubbles (prior to the rapid size increase near the end of thermal lifetimes; Fig. 5). Hereafter, we will refer to simulations based on the radius of their initial bubbles, and the environmental RH ("dry" corresponds to $\mathrm{RH}=42.5 \%$, and "moist" corresponds to $\mathrm{RH}=85 \%$ ).

\section{b. Comparison of theoretical expressions with axisymmetric simulations}

The following parameter values were used in the theoretical model from Part I for comparison with the axisymmetric simulations:

- We set $k^{2}=0.18$ and $P_{r}=1 / 3$, which are consistent with the values of these parameters used in the subgrid-scale mixing scheme for the simulations.

- We set $L=500 \mathrm{~m}$, which is the mixing length for parameterized subgrid-scale mixing in the axisymmetric simulations.

- Based on the simulated primary thermal radii in Fig. 5, $R$ values were set to the values shown in Table 1; however, simulations are referred to by the size of their initial bubbles. In the estimates of simulated thermal sizes, we neglected the rapid increases in thermal sizes at the end of their lifetimes. 

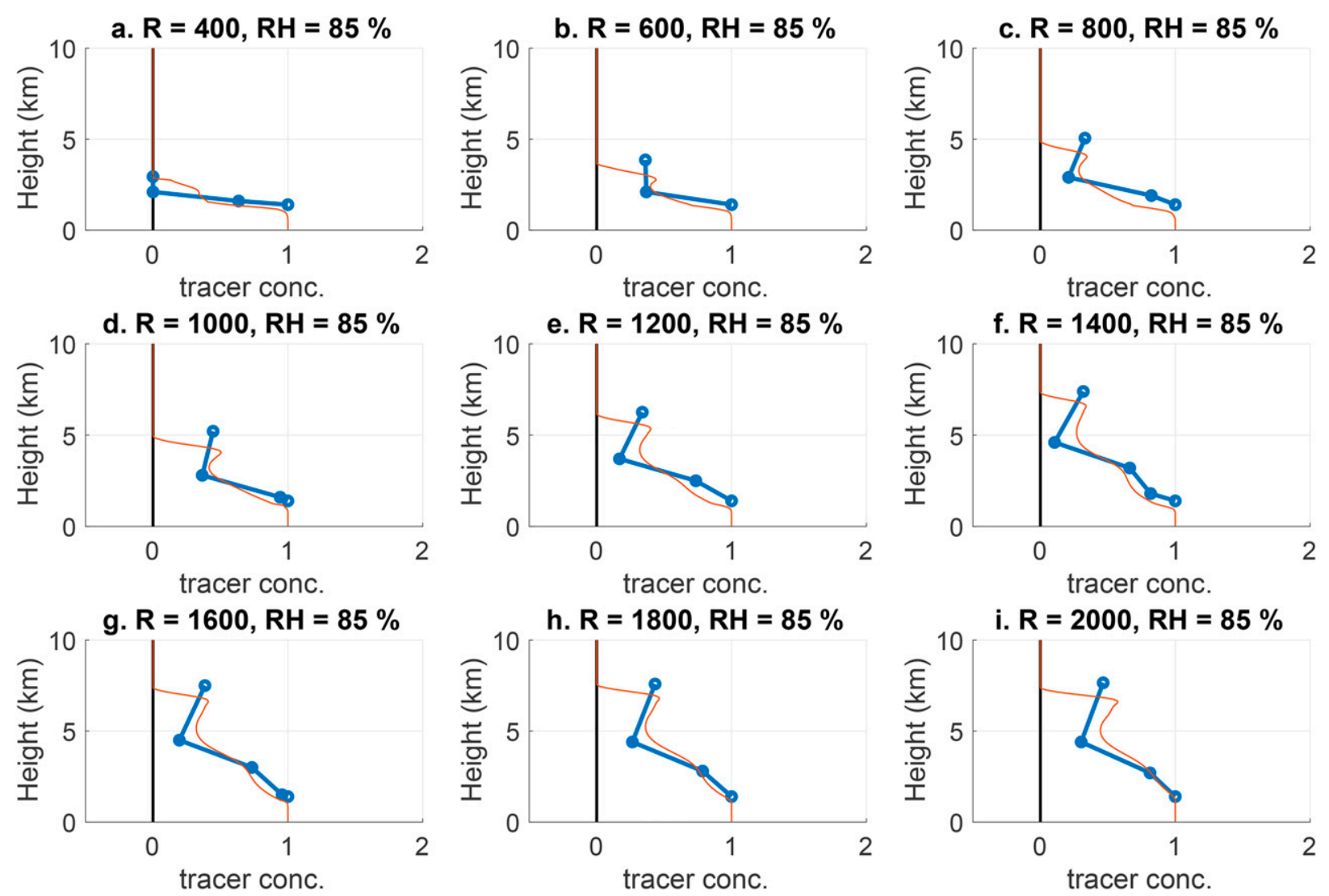

FIG. 9. As in Fig. 8, from the $\mathrm{RH}=85 \%$ runs.

- The environmental temperature and relative humidity profiles follow from those used for the simulations, as described in section 2. Adiabatic buoyancy $B_{\mathrm{AD}}$ is calculated from these profiles assuming pseudomoist adiabatic ascent above the level of free convection $(\sim 1400 \mathrm{~m})$, neglecting condensate loading.

- The following lower boundary conditions are used at the LFC: $C=1, B=0 \mathrm{~m} \mathrm{~s}^{-2}, w=0 \mathrm{~m} \mathrm{~s}^{-1}$.

The theoretical expressions from Part I provide solutions at the time when the top of the primary thermal is at height $z_{t}$. We calculate solutions for various thermal top height values as the updrafts evolve and the thermals rise (thermal top height values used in the plots are included in Table 1). Note that results are shown at slightly different thermal top height values for $C, B$, and $\varepsilon$ than for $w$ to highlight specific features discussed later (comparison of the theory and simulations is similar at other times).

The expressions from Part I are solved at $z_{t}, z_{b}, z_{m, 2}, z_{b, 2}$, etc., for the tracer and buoyancy values, and $z_{m}, z_{b}, z_{m, 2}, z_{b, 2}$, etc., for $w$. Each solution to the theoretical expressions for a given $R$ value corresponding to each simulation was compared to the vertical distribution of quantities at the center of the simulated axisymmetric updrafts ( $r=0$ in axisymmetric coordinates), and when the height of the uppermost $w$ maximum was equal to $z_{m}=z_{t}-R$. An exception to this approach is the comparison of theoretical and simulated $\varepsilon$. In this comparison, the simulated $\varepsilon$ values were calculated from the horizontal mass flux across the cloud updraft edge, normalized by the total vertical mass flux across the cloud at that height, as detailed in section 3b. Recall that a "cloudy updraft" volume is defined by grid points with $w>3 \mathrm{~m} \mathrm{~s}^{-1}$ and $q_{c}>10^{-5} \mathrm{~kg} \mathrm{~kg}^{-1}$. As detailed in Part I, the theoretical expression for $\varepsilon$ was determined by the dilution of a passive tracer at $r=0$ assuming a tracer value of 0 in the environment. Thus, by directly comparing the simulated and theoretical $\varepsilon$ we implicitly assume that entrainment and dilution are analogous. However, as pointed out by Romps (2010), "bulk" $\varepsilon$ values estimated from the dilution of a tracer can be up to a factor of 2 smaller than the "direct" entrainment calculation because of tracer heterogeneity in the environment. It is also assumed that the dilution of a tracer at $r=0$ is representative of the net entrainment across the updraft as a whole, at a given vertical level. This is reasonable given that the theoretical expressions well capture both the tracer values (and hence dilution) at $r=0$ and the $\varepsilon$ values from the simulations, as shown below.

Comparisons between theoretical and simulated $\varepsilon$ values (Figs. 6 and 7 ) show substantial qualitative similarities, though there are some quantitative differences. Local minima in $\varepsilon$ occurred at the top of the primary thermal and below it (near the top of the second thermal in simulations that produced a second thermal), in both the simulations and theoretical 
a. $\mathrm{R}=400, \mathrm{RH}=\mathbf{4 2 . 5} \%$

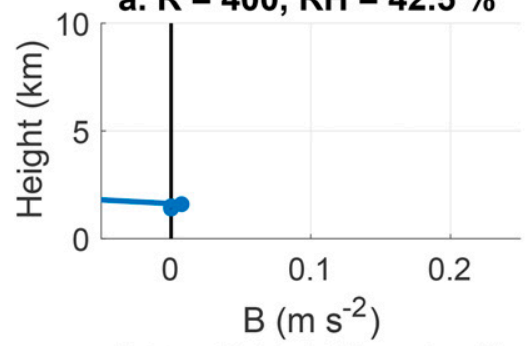

d. $R=1000, R H=42.5 \%$
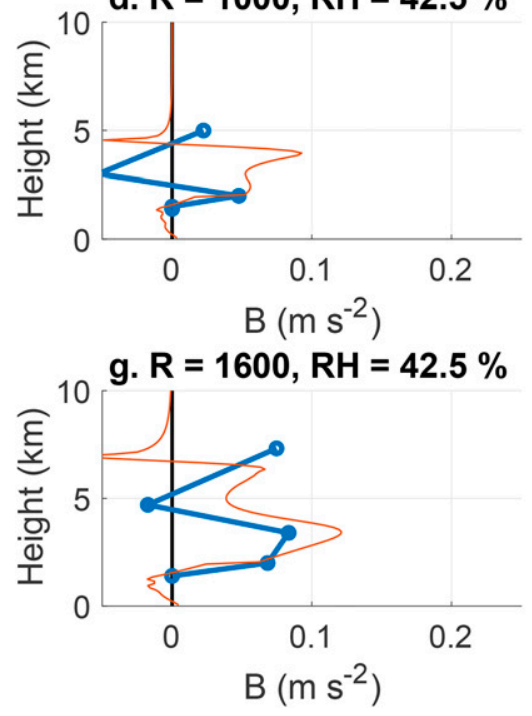

b. $\mathrm{R}=600, \mathrm{RH}=\mathbf{4 2 . 5} \%$

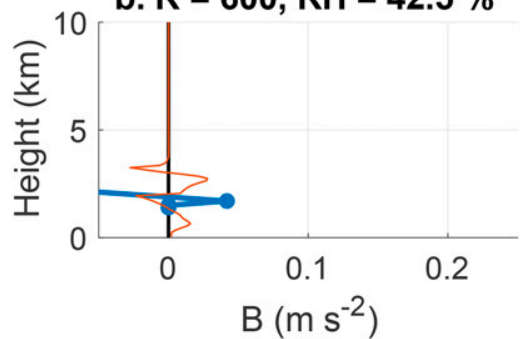

e. $R=1200, R H=42.5 \%$
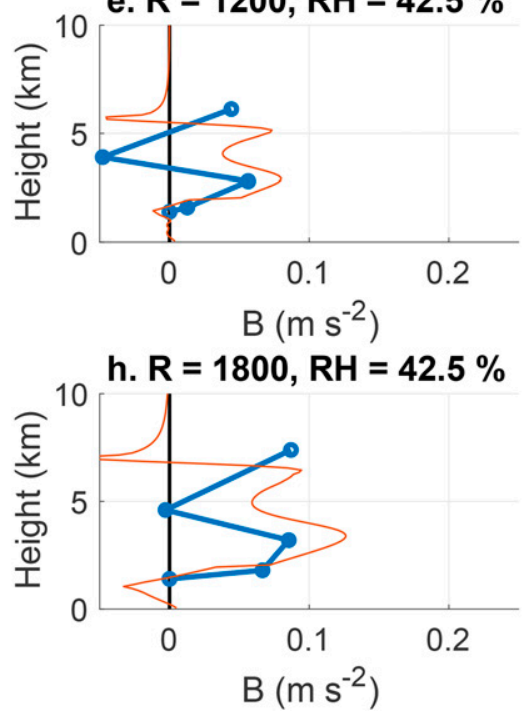
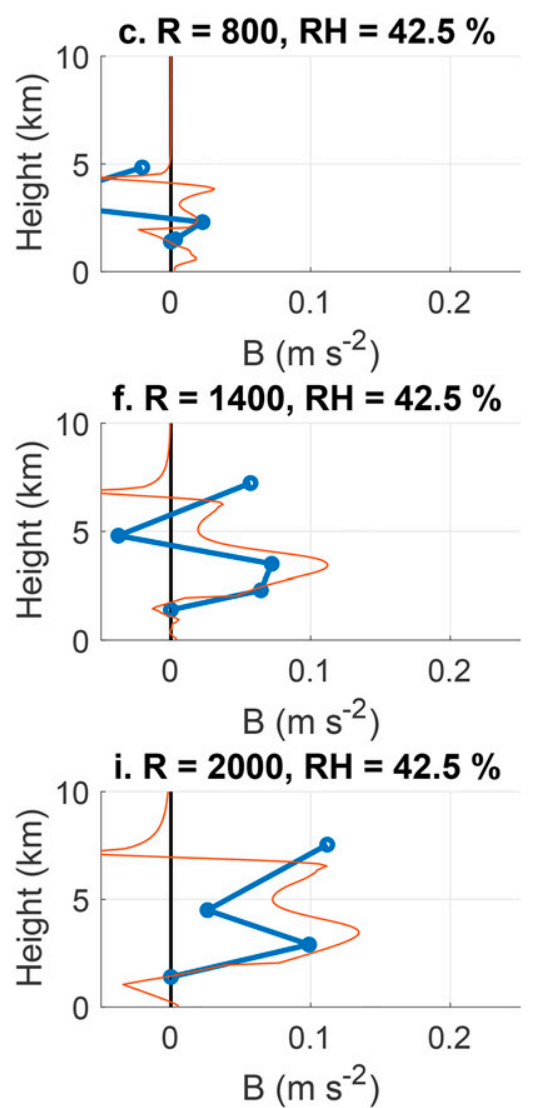

FIG. 10. As in Fig. 6, but for the buoyancy $\left(\mathrm{m} \mathrm{s}^{-2}\right)$ at $r=0$ from the RH $=42.5 \%$ runs.

expressions. Likewise, local maxima in $\varepsilon$ occurred at the bottoms of the primary and secondary thermals, where dynamic entrainment was locally enhanced associated with the "inward" branch of the thermals' toroidal circulations. Quantitative matches were best for intermediate radii, with the theoretical expressions underpredicting $\varepsilon$ for large radii and overpredicting $\varepsilon$ for small radii. It is possible that the assumption that properties of entrained air are equal to that of the background environment at a given height following the "bulk" approach contributes to the aforementioned quantitative discrepancies [for a discussion of biases introduced by the bulk approximation, see Romps (2010)].

Theoretical and simulated values of tracer $C$ also show similarities (Figs. 8 and 9 ). A local maximum in $C$ was typically present near the top of the primary thermal where there was a local minimum in $\varepsilon$. In contrast, a local minimum in $C$ was typically present at the bottom of the primary thermal where $\varepsilon$ was locally maximized. In both the theoretical and numerical solutions, values of $C$ generally decreased upward from the LFC to the bottom of the primary thermal, owing to the continuous action of entrainment and dilution at all heights, even where $\varepsilon$ was locally small. Patterns of $C$ were generally similar between the moist and dry runs for a given radii, which is consistent with the similar $\varepsilon$ between the moist and dry runs, and with the fact that $C$ is not directly influenced by the moisture content of the entrained air.

Unlike the updraft core $C$, the vertical distributions of core buoyancy (hereafter $B$ ) were quite different between the high-RH and low-RH runs, for given initial bubble radii. Many of the low-RH runs (Fig. 10) featured distinct double maxima in $B$-one near the top associated with the primary thermal, and one lower down associated with the secondary thermal. In contrast, the high-RH runs (Fig. 11) predominantly featured a maximum in $B$ associated with the primary thermal, and then a monotonic decrease in $B$ with decreasing height below this top maximum. Only the $1400-1800 \mathrm{~m}$ initial bubble radius runs had weak secondary local maxima in $B$ that were much less pronounced than in the lowRH runs.

Analysis of the theoretical expressions from Part I suggests that the more pronounced double maxima in $B$ in the dry runs were a result of the reduced condensation rates and evaporation associated with local maxima in fractional entrainment near the bottom of the primary thermal. Because fractional entrainment rates were fairly similar between the dry and moist runs for a given radius, the aforementioned buoyancy differences mainly resulted from the lower $\mathrm{RH}$ of the entrained air in the dry runs. Indeed, the differences in $B$ between the moist and dry axisymmetric runs were well 

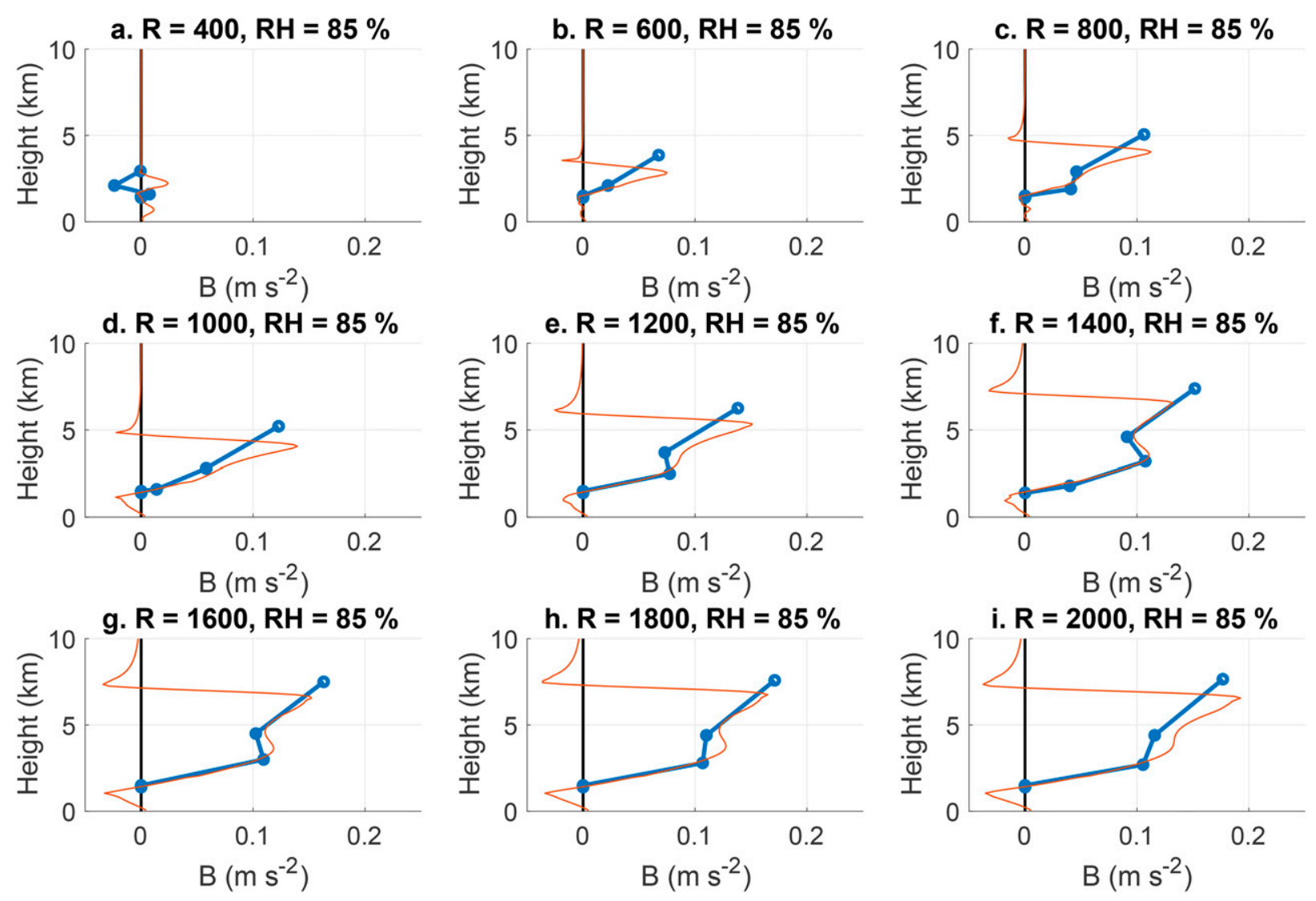

FIG. 11. As in Fig. 10, from the $\mathrm{RH}=85 \%$ runs.

captured by the theoretical expressions, which affirms the conclusion from Part I.

Similar differences in the core $w$ profiles are present between the low-RH and high-RH runs for a given $R$. Pronounced double maxima in the vertical profiles of $w$ were present in the dry runs (Fig. 12), whereas a more or less monotonic increase in $w$ with height below the level of maximum $w$ was present in the moist runs (Fig. 13). Again, these differences in the profiles were well captured by the theoretical expressions.

Overall, the main similarities and differences between the moist and dry axisymmetric runs were captured by the theoretical model. Values of $\varepsilon$ and $C$ were similar between the moist and dry runs, whereas pronounced double maxima in the vertical profiles of $B$ and $w$ were present in the dry runs, but largely absent in the moist runs. Trends generally showed a succession of discrete thermals in the dry runs, whereas an evolution toward a sustained plume of positive $B$ and $w$ extending through much of the troposphere occurred in the moist runs. Likewise, trends in the simulations showed a progression from isolated thermals at small radii, to thermal chains at larger radii in the dry environment, to a plume or starting plume structure at larger radii in the moist environment. This behavior with respect to radius and environmental relative humidity is consistent with the theoretical model.

\section{c. Turbulent $3 D$ simulations}

Turbulence in the 3D runs produced notably more complex updraft behavior than in the axisymmetric runs, as expected. All runs featured the flow signatures of thermals, including well-defined toroidal circulations (e.g., see the flow vectors in Figs. 14-17). Side-by-side comparisons of the moist and dry runs reveal behavior that is consistent with both theory and the axisymmetric simulations. In the runs with $R=500 \mathrm{~m}$, a single thermal was produced in the dry run (Figs. 14a,c,e), and a dominant primary thermal was produced in the moist run with hints of the development of a secondary thermal below the large initial thermal (Figs. 14b,d,f). This supports the theoretical model, which suggests that isolated thermals are produced in dry environments at small radii, and the beginnings of a thermal chain structure should develop in moist environments at small radii.

In the runs with an initial $R$ of $1000 \mathrm{~m}$, multiple distinct thermals were produced in both the dry (Figs. 15a,c,e) and moist runs (Figs. 15b,d,f). The dry runs, however, had more distinct regions of low vertical velocity between individual rising thermals than in the moist runs (see annotation in Fig. 15c), whereas the moist runs featured a comparatively continuous region of rising motion through the cloud depth. Similar differences were present between the dry (Figs. 16a,c,e) and moist (Figs. 16b,d,f) $1500 \mathrm{~m}$ runs, and 

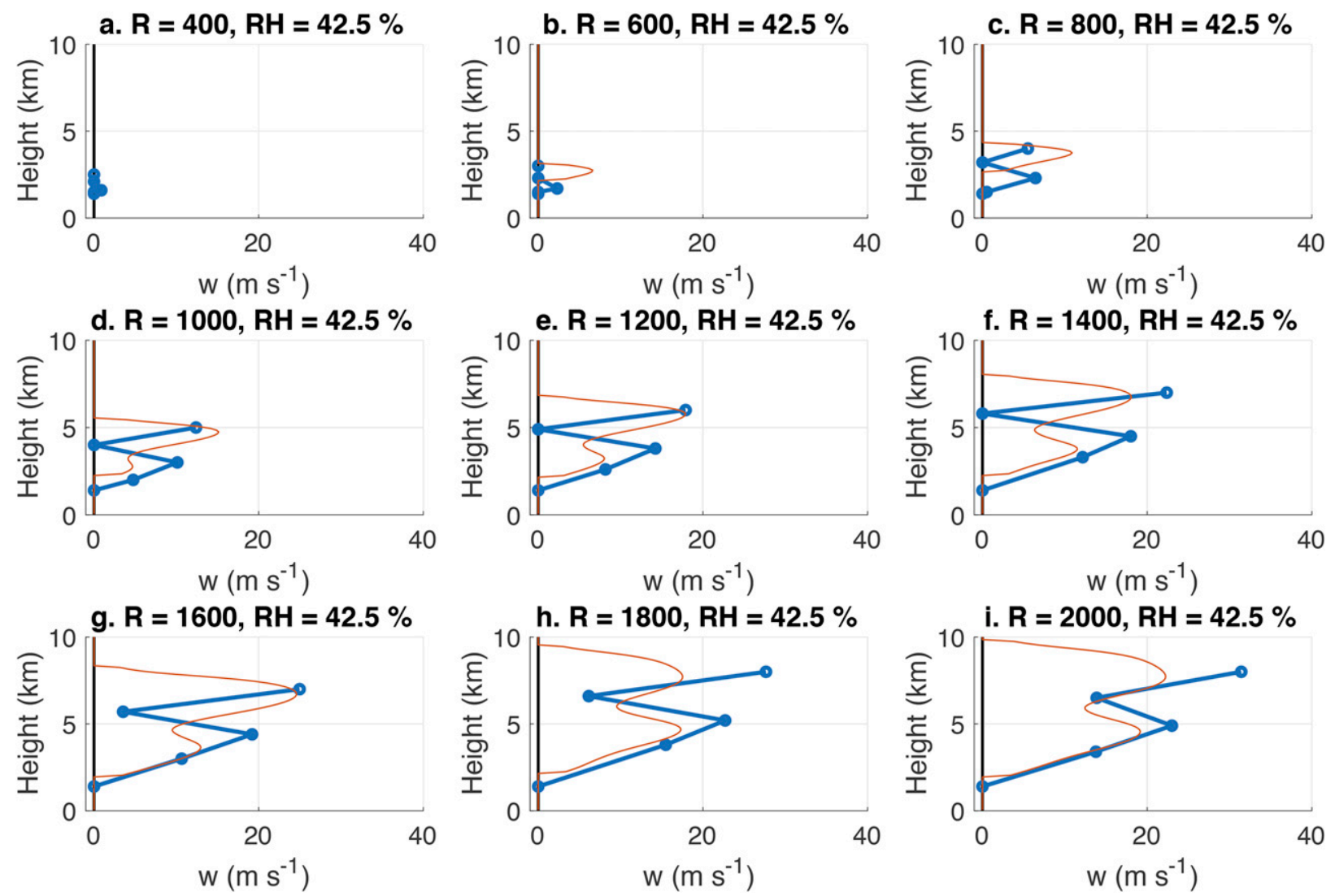

FIG. 12. As in Fig. 6, but for vertical velocity $\left(\mathrm{m} \mathrm{s}^{-1}\right)$ at $r=0$ from the $\mathrm{RH}=42.5 \%$ runs.

between the dry (Figs. 17a,c,e) and moist (Figs. 17b,d,f) $2000 \mathrm{~m}$ runs. These differences are again consistent with the axisymmetric runs and theoretical expressions.

Distinct rising "streaks" of relatively large horizontalmaximum $w$ associated with individual thermals are evident in time-height diagrams, consistent with the thermal chain structure (Fig. 18). There was more prolonged ascent and deeper layers of monotonically increasing horizontalmaximum $w$ in the moist runs than in the dry runs, similar to the axisymmetric simulations (cf. Fig. 18 to Figs. 1 and 3). For example, after ascent and decay of the primary ascending thermal (after about $17 \mathrm{~min}$ ), the $2000 \mathrm{~m}$ initial bubble radius moist $3 \mathrm{D}$ run produced deep ascent with monotonically increasing horizontal-maximum $w$ up to heights of 7-9 km (Fig. 18f). In contrast, the $2000 \mathrm{~m}$ dry 3D run produced three distinct additional thermal-like structures in the lower to middle troposphere (seen by "streaks" of large horizontal-maximum $w$ ) after ascent and decay of the primary thermal (Fig. 18e). Also consistent with the axisymmetric runs, the appearance of secondary thermal structures in the dry 3D runs occurred earlier and at lower altitudes than in the moist 3D runs, for a given initial bubble radius. Interestingly, the moist $3 \mathrm{D}$ runs overall featured more pronounced secondary thermal-like circulations than the axisymmetric moist runs (cf. Figs. 17d,f and 4). We hypothesize that turbulence promotes a breakdown of large initial bubbles into smaller thermals with more distinctive toroidal circulations. However, a thorough investigation of the role of turbulence in promoting thermal-like circulations within updrafts is beyond the scope of this paper, and left to future work. Also note that individual thermals in the chain-like LES updrafts are not vertically aligned because of turbulence-induced horizontal drift, unlike in the axisymmetric simulations, but the thermals do occur in succession.

Despite the turbulent nature of the flow in the $3 \mathrm{D}$ runs, the time evolution of fractional entrainment rate was dominated by "streaks" of relatively large $\varepsilon$ near the bottom individual ascending thermals with vertical scales of $1-2 \mathrm{~km}$, particularly in the dry simulations (Fig. 18). This affirms the result from the theoretical model that the vertical profile of entrainment is largely modulated by organized inflow near the base of individual thermals that have radii similar to the updraft as a whole $(1-2 \mathrm{~km})$. The time evolution of $\delta$, on the other hand, was dominated by large detrainment near the end of thermals' ascent paths (Fig. 19), and between successive thermals in the dry runs (Figs. 19a,c,e). The presence of larger fractional detrainment between individual thermals in the dry runs, than in the moist runs, further illustrates the tendency for more discrete thermal-like structures with well defined inflow (entrainment) and outflow (detrainment) regions in the dry runs. 

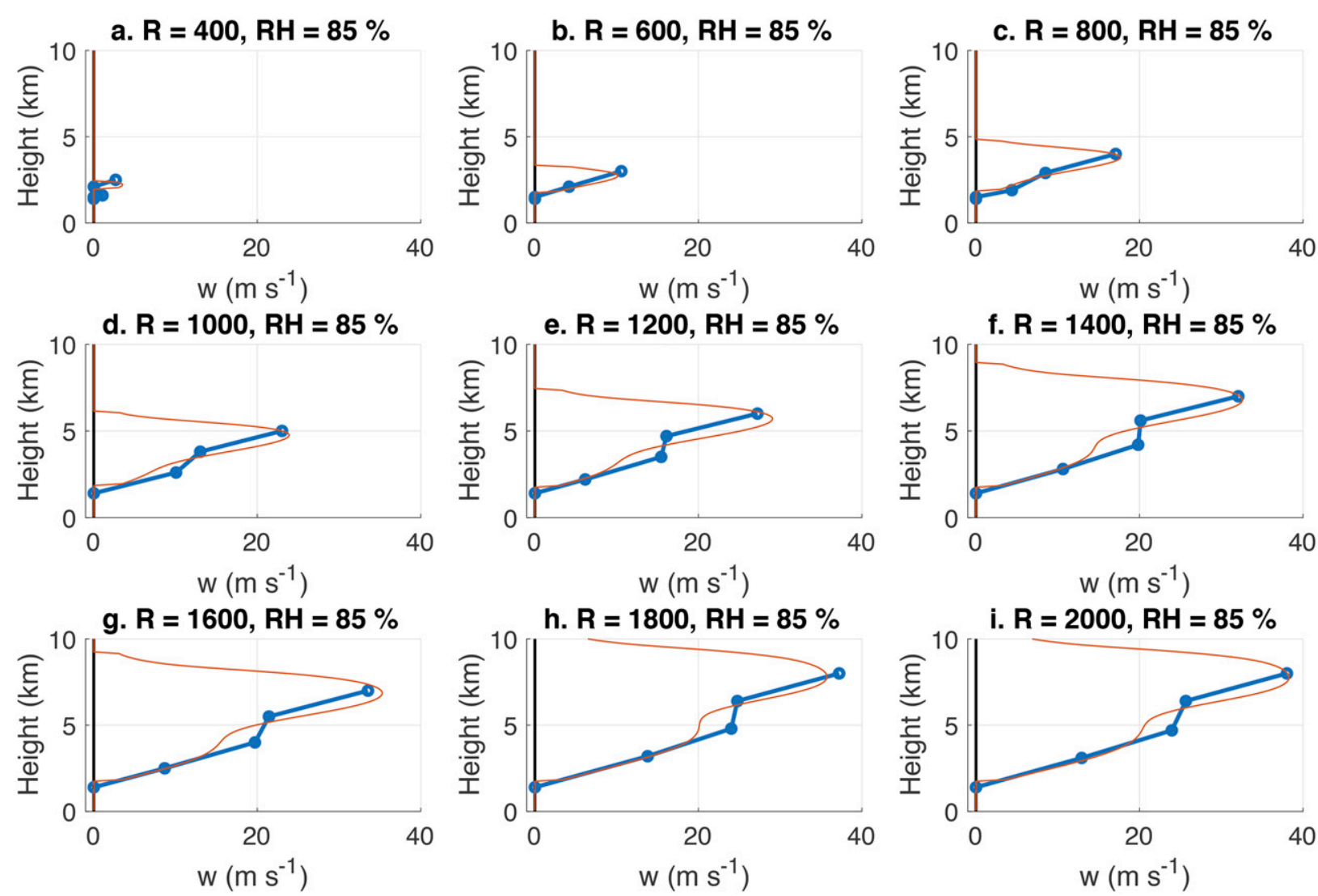

FIG. 13. As in Fig. 12, from the RH $=85 \%$ runs.

The vertical structure of $\varepsilon$ and horizontal-maximum $w$ in the 3D runs is further illustrated in Fig. 20, which shows profiles from the 1000 and $1500 \mathrm{~m}$ initial bubble runs at $12.5 \mathrm{~min}$. Included in Fig. 20 are profiles of $\varepsilon$ and $w$ from the theoretical expressions calculated using the parameters from section $3 \mathrm{~b}$, except $R$ is assumed to be equal to the initial bubble radius from the simulations for simplicity and $L=R / 3$ (following Part I). Local maxima of $\varepsilon$ in the $3 \mathrm{D}$ runs generally coincided with local minima in $w$, consistent with the behavior of the theoretical model. A deep layer of near-monotonically increasing horizontal-maximum $w$ is seen in the $1500 \mathrm{~m}$ bubble moist 3D run (Fig. 20b), but in the corresponding dry $3 \mathrm{D}$ run (Fig. 20a) $w$ was substantially reduced below the primary thermal (whose center is near $8 \mathrm{~km}$ ), and there were two distinct local $w$ minima coinciding with maxima in $\varepsilon$ (Fig. 20a). Interestingly, the magnitude of $\varepsilon$ was similar among the dry and moist runs, suggesting that the differences between these runs are mainly because of the low RH of the entrained environmental air in the dry runs. This behavior is also consistent with the axisymmetric simulations and theory. Similar differences are evident in the dry and moist $1500 \mathrm{~m}$ initial bubble runs (Figs. 20c,d), with a pronounced local secondary $w$ maximum in the dry run below the primary thermal but no distinct secondary $w$ maximum in the moist run. There are notable quantitative differences between the theoretical results and those from the $3 \mathrm{D}$ runs. The peaks in $\varepsilon$ are generally much smaller from the theoretical model. These differences likely arise, at least in part, from the simple approximations to vertical $w$ gradients made when deriving the theoretical $\varepsilon$ at the thermal bottom and below (see Part I) and the bulk-plume assumption invoked to derive the theoretical $\varepsilon$ that properties of entrained air are the same as the far-field background environment. Local $w$ minima from the theoretical expressions are also much smaller than in the $3 \mathrm{D}$ runs for the dry environment. There are several possible explanations for this difference, including the aforementioned bulk-plume assumption, neglect of vertical mixing in the theoretical model, and neglect of dynamic pressure forcing below the primary thermal (see Part I).

\section{Summary, discussion, and conclusions}

Previous numerical modeling and observational studies show that the fundamental structure of deep convection often consists of a succession of rising thermals (e.g., Raymond and Blyth 1989; Blyth and Latham 1993; Damiani et al. 2006; Moser and Lasher-Trapp 2017; Peters et al. 2019), which we call a "thermal chain." This structure is distinct from the assumed structure for convective updrafts in conceptual models and cumulus parameterizations.

As a step toward improving understanding of the thermal chain structure, this study used numerical simulations to 
Time: $10.5 \mathrm{~min}$
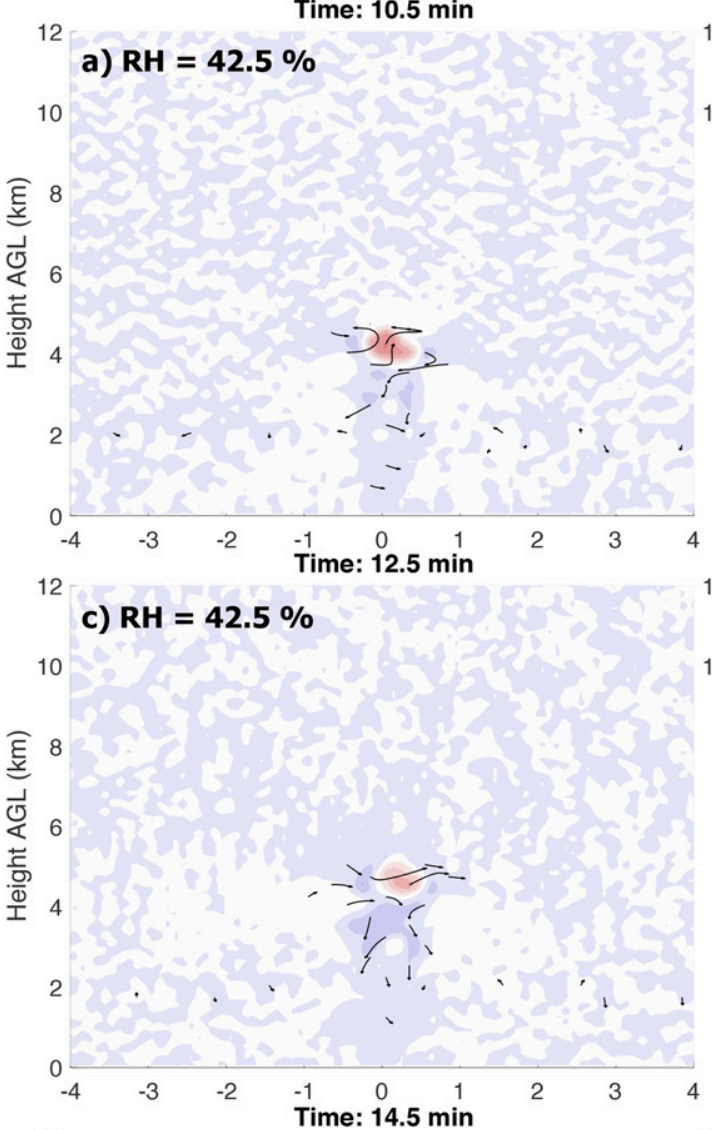

12
Time: $10.5 \mathrm{~min}$

b) $\mathbf{R H}=\mathbf{8 5} \%$
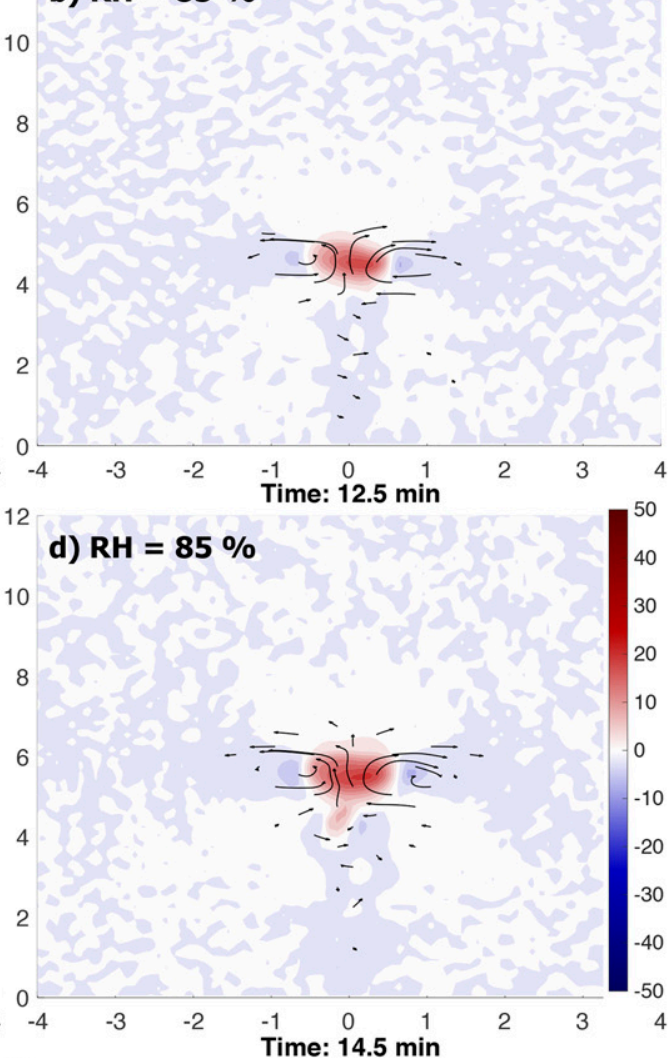

e) $\mathbf{R H}=\mathbf{4 2 . 5} \%$
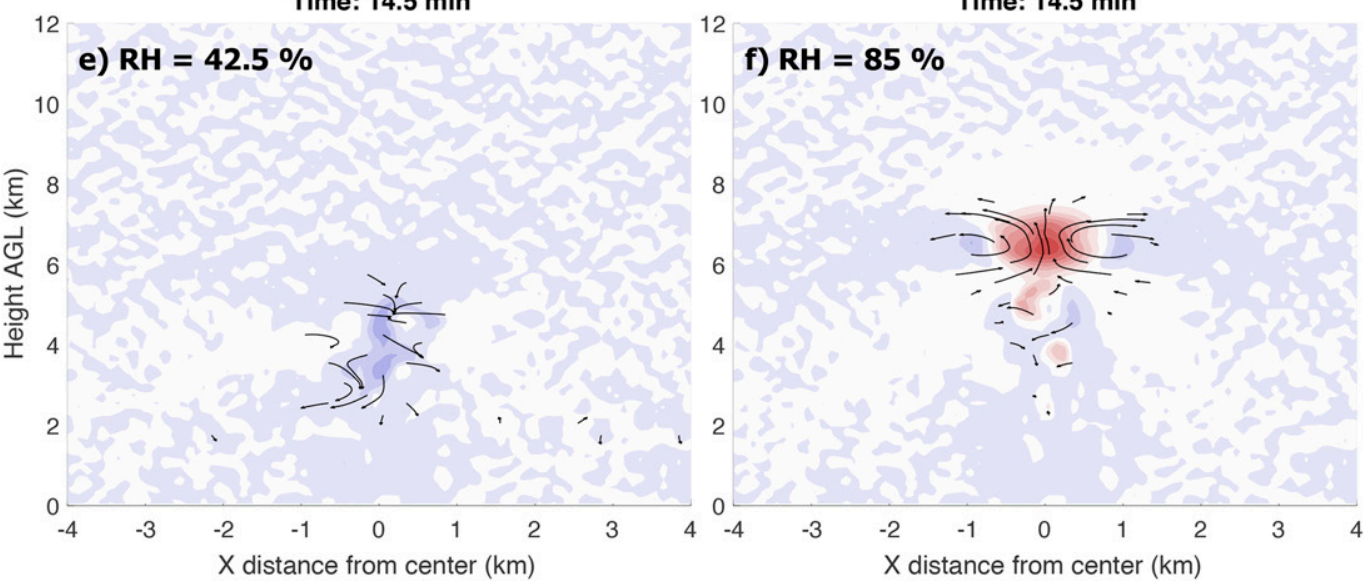

FIG. 14. Vertical cross sections along the $x$ axis from 3D simulations of vertical velocity (shading, $\mathrm{m} \mathrm{s}^{-1}$ ) and flow vectors (black arrows) from the 3D simulations with an initial bubble radius $(R)$ of $500 \mathrm{~m}$. Columns show the (a),(c),(e) $\mathrm{RH}=42.5 \%$ and (right) $\mathrm{RH}=85 \%$ runs. Rows show results valid at (top) 10.5 , (middle) 12.5 , and (bottom) $14.5 \mathrm{~min}$.

address why this structure is seemingly prevalent in deep moist convection. From the theoretical expressions obtained in Part I, thermal chains were hypothesized to arise from interactions between updraft flow structure and entrainment of dry environmental air. In this mechanism, organized inflow below the height of maximum $w$ associated with the primary ascending thermal leads to enhanced entrainment of dry environmental air. These dry-air intrusions reduce condensation rates, increase evaporation, erode buoyancy, and cause a breakdown of the updraft into a succession of rising thermals. Entrainment of dry air is therefore essential to the development of chainlike cumulus updrafts. From the theoretical model in Part I, 
Time: $\mathbf{1 0 . 5} \mathrm{min}$
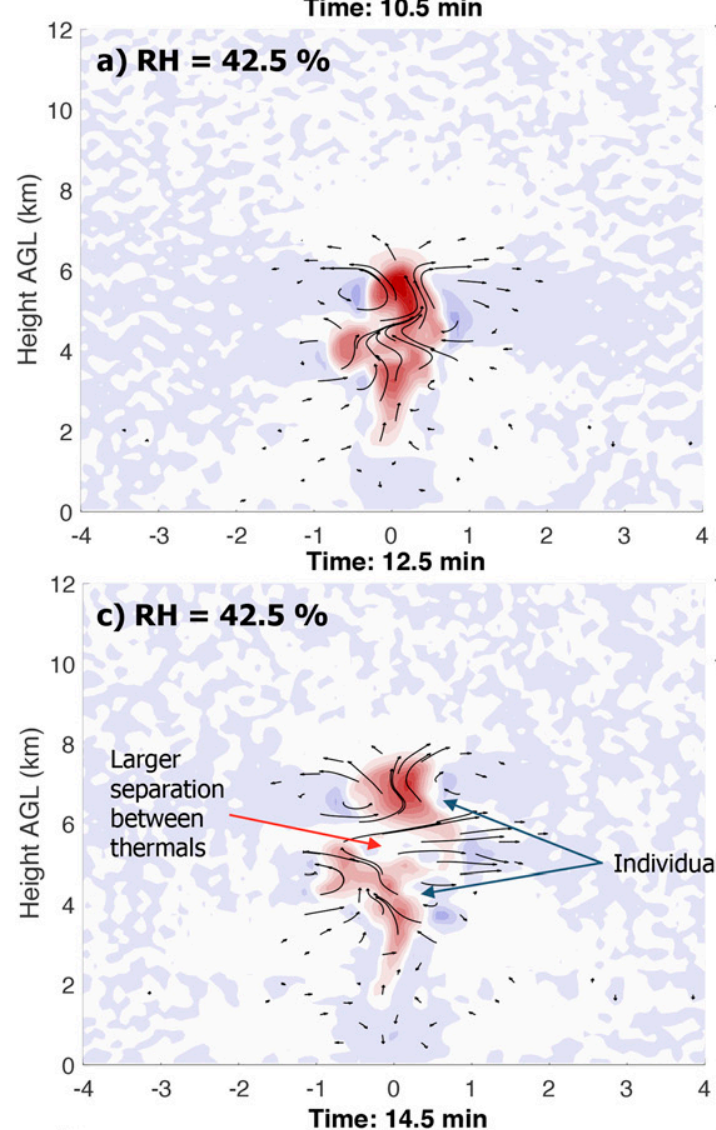

12
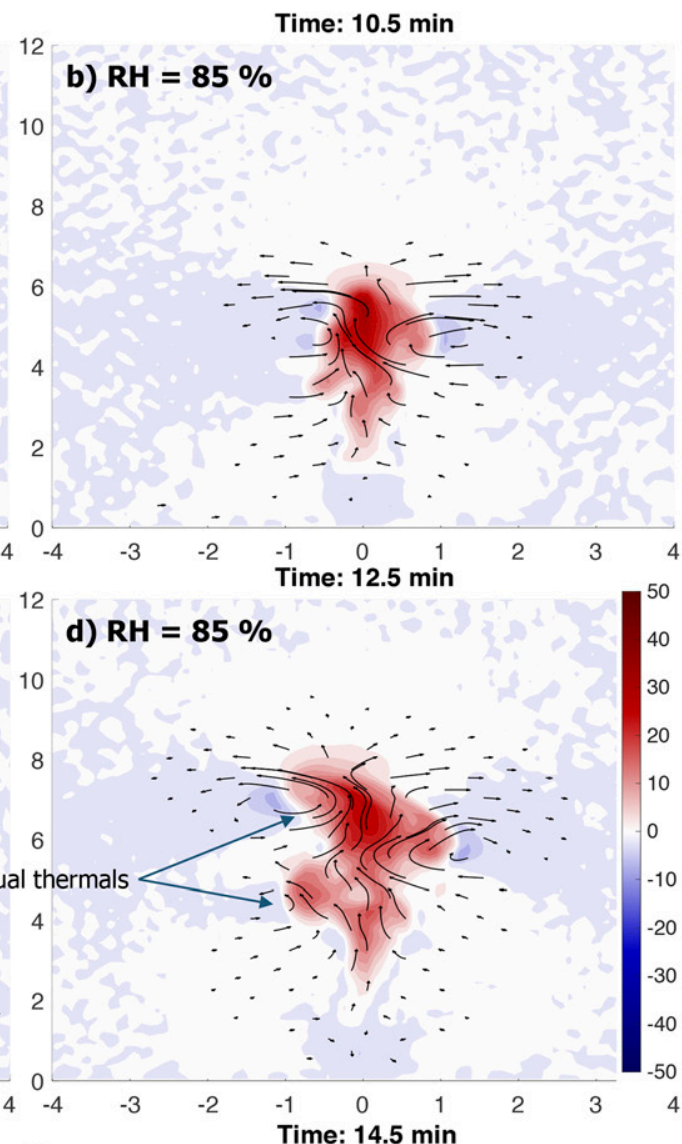

f) $\mathbf{R H}=\mathbf{8 5} \%$
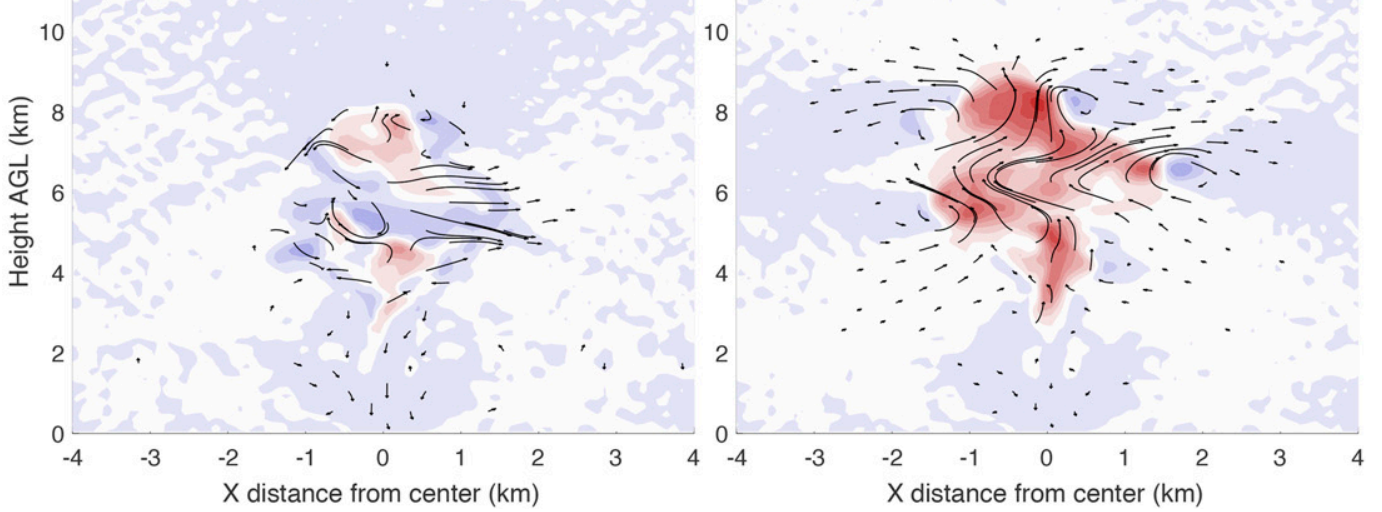

FIG. 15. As in Fig. 14, but for the runs with an initial bubble radius of $1000 \mathrm{~m}$.

for clouds with small initial widths (e.g., radius $<800 \mathrm{~m}$ ) and dry middle-tropospheric environments (e.g., $\mathrm{RH}<$ $50 \%$ ), dry-air entrainment is sufficient to entirely erode the updraft region below the primary ascending thermal, leading to isolated thermals. For clouds with intermediary initial widths and dry middle-tropospheric environments (e.g., radius $>800 \mathrm{~m}$ ), dry-air entrainment leads to the development of thermal chains. For moist middletropospheric environments and moderate-to-large cloud widths, updrafts evolve toward continuous plumes of rising air.

A series of axisymmetric simulations of deep convection were run for this study to test these ideas, wherein the radius of the warm bubble used to initiate convection and the middletropospheric relative humidity were varied. Results from these simulations strongly support the above hypothesis, in terms of the dependency of cloud behavior and structure on initial cloud radius and middle-tropospheric relative humidity. A set of 

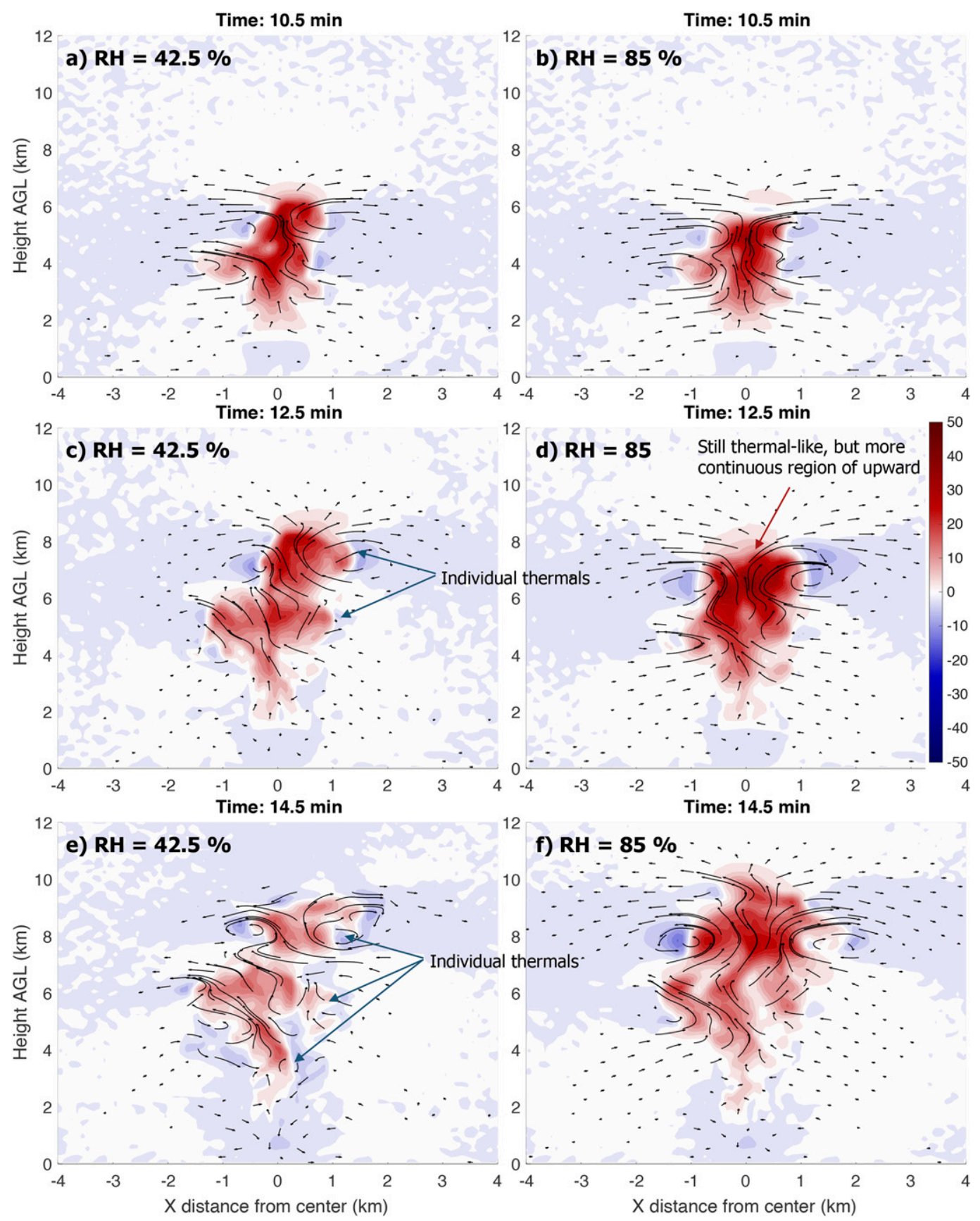

FIG. 16. As in Fig. 14, but for the runs with an initial bubble radius of $1500 \mathrm{~m}$.

turbulent 3D runs were also performed with similar initial bubble size and moisture variations. Although these 3D runs were much more complicated owing to their explicit representation of turbulence, they showed qualitatively consistent behavior with the axisymmetric runs and theoretical expressions. These results suggest that turbulence is not itself a critical feature explaining the occurrence of thermal chain updrafts, though individual thermal-like circulations were more distinct in the turbulent 3D moist environment runs than in the corresponding nonturbulent axisymmetric runs. Overall, the axisymmetric and 3D runs showed that locally large fractional entrainment rates near the bottom of individual thermals were associated with local minima in updraft core buoyancy and vertical velocity. These results support the conclusion from Part I that entrainment is dominated by inflowing environmental air associated with thermals' toroidal 

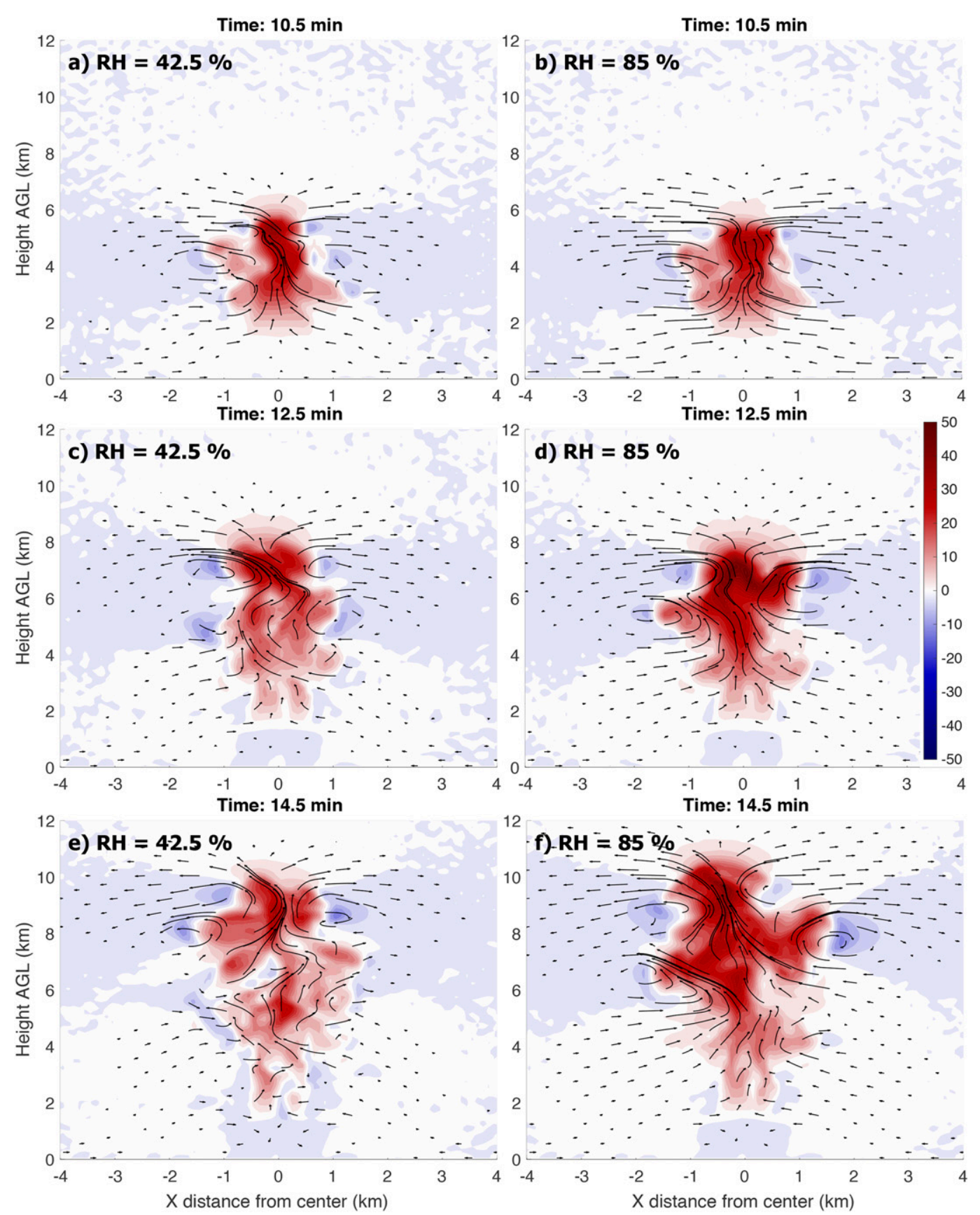

FIG. 17. As in Fig. 14, but for the runs with an initial bubble radius of $2000 \mathrm{~m}$.

circulations, substantially contributing to overall cloud dilution while thermal core regions can remain relatively undilute.

In this study, we examined the entrainment behavior of individual cumulus clouds to gain process-level understanding, particularly by relating variability in entrainment rates to updraft structure. We do not attempt to draw broader conclusions from a statistical analysis of fractional entrainment rates and various convective parameters because the simulations here were conducted over a limited range of atmospheric environments. However, the entrainment dependencies generally agree with those shown in Fig. 13 of Part I (not shown).

A caveat to the modeling approaches used in this study is that they all relied on warm-bubble convective initialization. In nature, a variety of comparatively complex processes lead to the initial development and evolution of cumulus clouds, such as boundary layer turbulence, mesoscale atmospheric 
a. $\mathrm{R}=1000, \mathrm{RH}=42.5 \%$
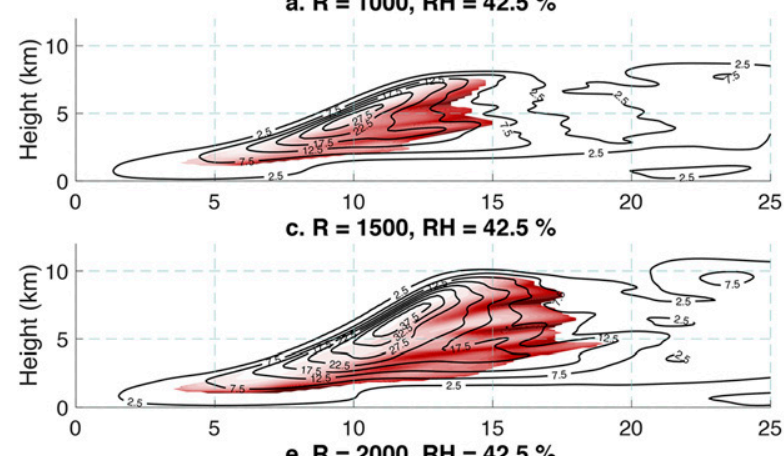

e. $R=2000, R H=42.5 \%$

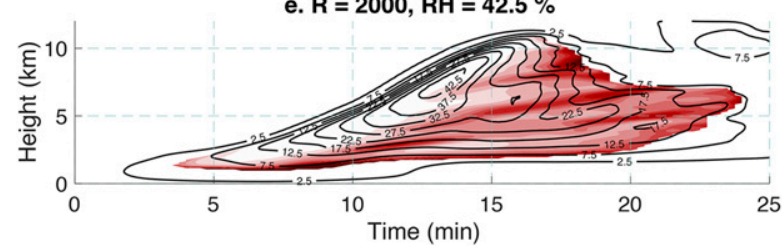

b. $R=1000, \mathrm{RH}=85 \%$
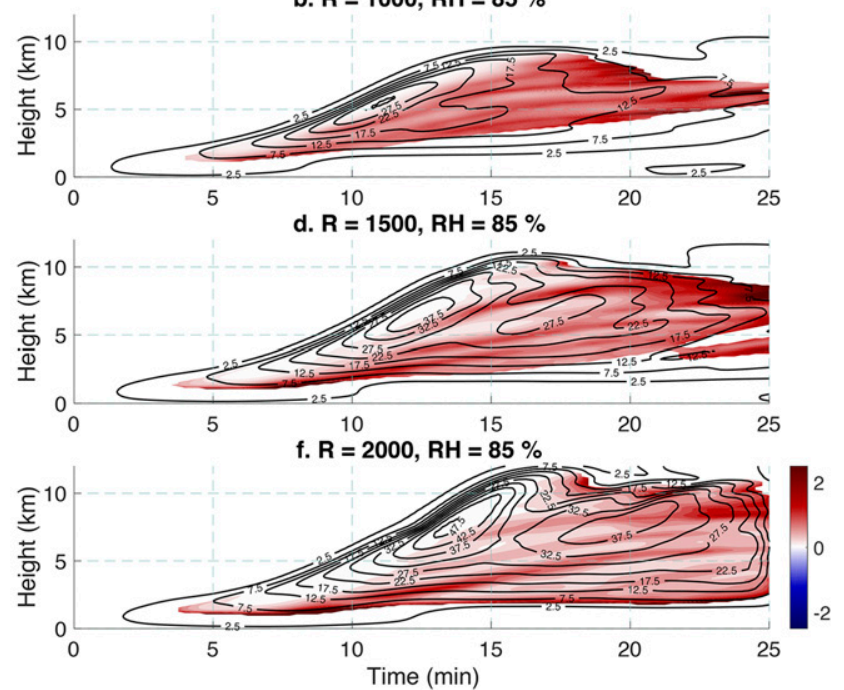

FIG. 18. Time-height diagram of fractional entrainment rate $\varepsilon$ (shading, $\mathrm{km}^{-1}$ ) and level-maximum vertical velocity (black contours at intervals of $5 \mathrm{~m} \mathrm{~s}^{-1}$, starting at $2.5 \mathrm{~m} \mathrm{~s}^{-1}$ ). Columns show the $3 \mathrm{D}$ simulation with (a),(c),(e) $\mathrm{RH}=42.5 \%$ and (b), (d),(f) $\mathrm{RH}=85 \%$. Initial bubble radii are (top) 1000, (middle) 1500 , and (bottom) $2000 \mathrm{~m}$.

boundaries, and terrain variability. The inclusion of such complexity in LES requires large domains (e.g., of order $100 \mathrm{~km}$ horizontal dimensions), long spinup time to produce a realistic deep-convective "scene," realistic forcing from surface fluxes and/or large-scale atmospheric tendencies, and possibly initial and lateral boundary conditions provided by analyses and more sophisticated microphysics schemes that include ice and precipitation. Future work should investigate the theory developed in our study using these more resourceintensive LES configurations.
As was discussed in Part I, a potentially substantial influencing factor on the thermal chain structure is vertical wind shear, which was neglected in this study. The role of shear on thermal ascent rates was studied by Peters et al. (2019); however, that study did not specifically investigate how shear influences how thermals develop and the dependency of their behavior on initial cloud radius and relative humidity. Future work should incorporate the effects of vertical wind shear into the theory, given that most convection around the world occurs in environments with at least some shear.
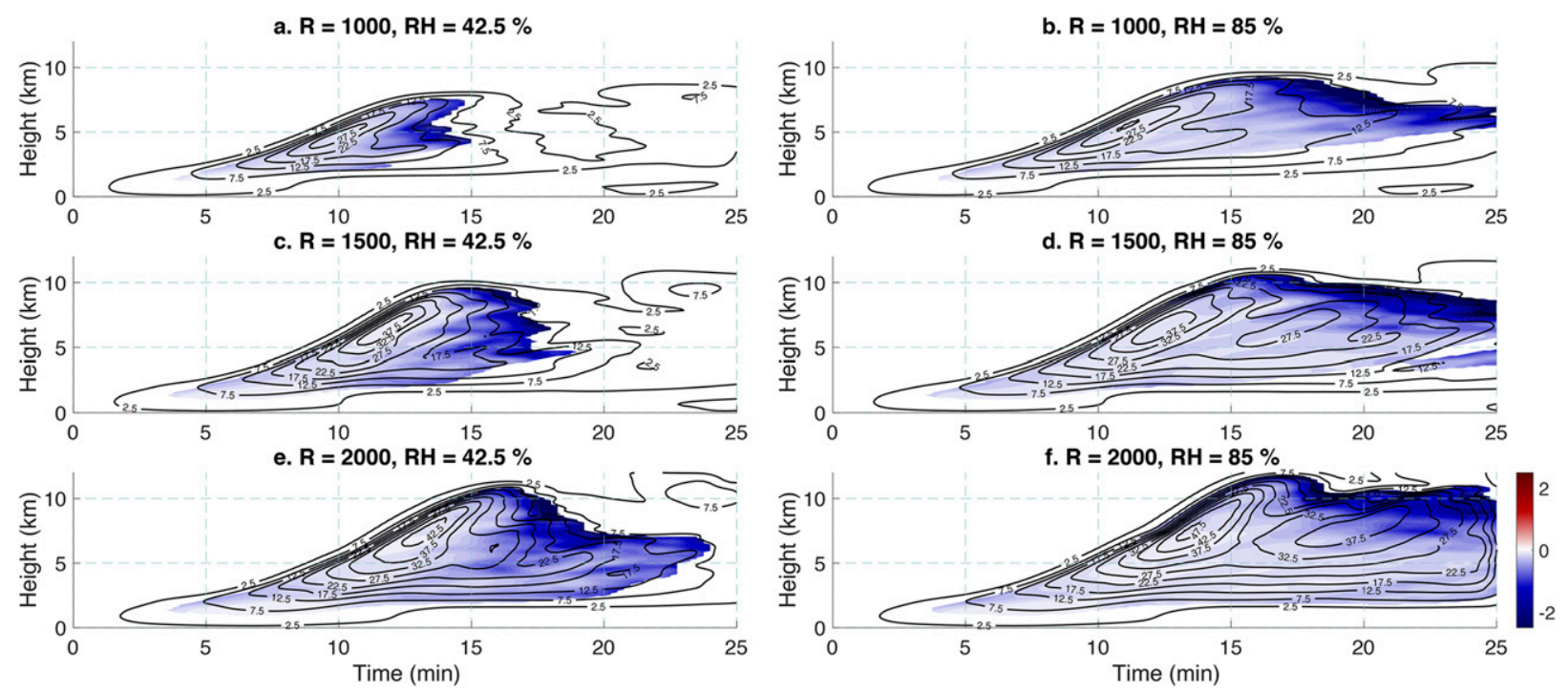

FIG. 19. As in Fig. 18, but with fractional detrainment rate $\delta$ shaded $\left(\mathrm{km}^{-1}\right)$. 

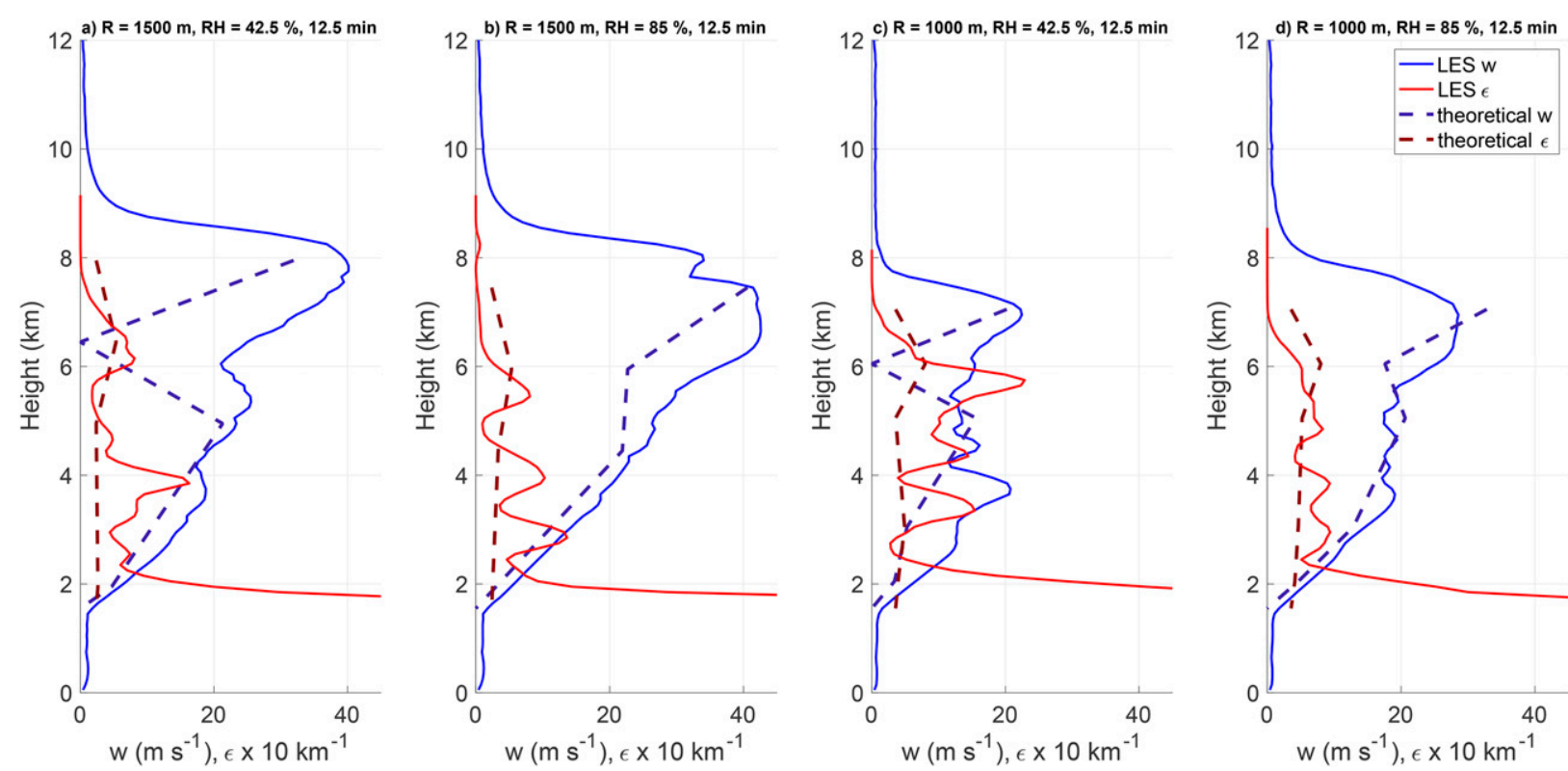

FIG. 20. Comparisons of horizontal-maximum vertical velocity $w\left(\mathrm{~m} \mathrm{~s}^{-1}\right)$ and fractional entrainment rate $\varepsilon\left(\times 10, \mathrm{~km}^{-1}\right)$ from the 3D runs (solid blue and solid red lines, respectively) with $w$ and $\varepsilon$ from the theoretical model (blue dashed and red dashed lines, respectively, same units). All comparisons are $12.5 \mathrm{~min}$ into the simulations. Run details are listed in the panel titles. In the theoretical model, we used the initial bubble radius in the simulation and $L=R / 3$ (as in Part I).

Acknowledgments. This material is based on work supported by the National Center of Meteorology, Abu Dhabi, UAE, under the UAE Research Program for Rain Enhancement Science and the U.S. Department of Energy Atmospheric System Research (Grants DE-SC0016476, DE-SC0020104, and DE-SC0000246356). J. Peters's efforts were also partially supported by the National Science Foundation Grant AGS1841674. A. Varble was supported by the U.S. Department of Energy Office of Science Biological and Environmental Research as part of the Atmospheric System Research program. Pacific Northwest National Laboratory is operated by Battelle for the U.S. Department of Energy under Contract DE-AC05-76 RLO1830. W. Hannah's work was performed under the auspices of the U.S. DOE by Lawrence Livermore National Laboratory under Contract DE-AC52-07 NA27344. S. Giangrande's work was performed under the auspices of Brookhaven Science Associates, LLC, under Contract DE-SC0012704 with the U.S. DOE. The publisher by accepting the paper for publication acknowledges that the U.S. Government retains a nonexclusive, paid-up, irrevocable, worldwide license to publish or reproduce the published form of this paper, or allow others to do so, for U.S. Government purposes. We would like to acknowledge high-performance computing support from Cheyenne (doi:10.5065/D6RX99HX) provided by NCAR's Computational and Information Systems Laboratory. The National Center for Atmospheric Research is sponsored by the National Science Foundation.

\section{REFERENCES}

Blyth, A. M., and J. Latham, 1993: Development of ice and precipitation in New Mexican summertime cumulus clouds. Quart. J. Roy. Meteor. Soc., 119, 91-120, https://doi.org/10.1002/qj.49711950905.
Bryan, G. H., and J. M. Fritsch, 2002: A benchmark simulation for moist nonhydrostatic numerical models. Mon. Wea. Rev., 130, 2917-2928, https://doi.org/10.1175/1520-0493(2002)130<2917: ABSFMN $>2.0 . C O ; 2$.

Damiani, R., and G. Vali, 2007: Evidence for tilted toroidal circulations in cumulus. J. Atmos. Sci., 64, 2045-2060, https:// doi.org/10.1175/JAS3941.1.

— _ _ a and S. Haimov, 2006: The structure of thermals in cumulus from airborne dual-Doppler radar observations. J. Atmos. Sci., 63, 1432-1450, https://doi.org/10.1175/JAS3701.1.

De Rooy, W. C., and Coauthors, 2013: Entrainment and detrainment in cumulus convection: An overview. Quart. J. Roy. Meteor. Soc., 139, 1-19, https://doi.org/10.1002/qj.1959.

Emanuel, K. A., 1994: Atmospheric Convection. Oxford University Press, 588 pp.

Hannah, W. M., 2017: Entrainment versus dilution in tropical deep convection. J. Atmos. Sci., 74, 3725-3747, https://doi.org/ 10.1175/JAS-D-16-0169.1.

Hernandez-Deckers, D., and S. C. Sherwood, 2016: A numerical investigation of cumulus thermals. J. Atmos. Sci., 73, 4117-4136, https://doi.org/10.1175/JAS-D-15-0385.1.

Khairoutdinov, M., S. K. Krueger, C.-H. Moeng, P. A. Bogenshultz, and D. A. Randall, 2009: Large-eddy simulation of maritime deep tropical convection. J. Adv. Model. Earth Sys., 1 (4), https://doi.org/10.3894/JAMES.2009.1.15.

Klemp, J. B., and R. B. Wilhelmson, 1978: Simulations of right- and left-moving storms produced through storm splitting. J. Atmos. Sci., 35, 1097-1110, https://doi.org/10.1175/1520-0469(1978) 035<1097:SORALM > 2.0.CO;2.

Lebo, Z. J., and H. Morrison, 2015: Effects of horizontal and vertical grid spacing on mixing in simulated squall lines and implications for convective strength and structure. Mon. Wea. Rev., 143, 4355-4375, https://doi.org/10.1175/MWR-D15-0154.1. 
McKim, B., N. Jeevanjee, and D. Lecoanet, 2020: Buoyancy-driven entrainment in dry thermals. Quart. J. Roy. Meteor. Soc., 146, 415-425, https://doi.org/10.1002/qj.3683.

Morrison, H., 2017: An analytic description of the structure and evolution of growing deep cumulus updrafts. J. Atmos. Sci., 74, 809-834, https://doi.org/10.1175/JAS-D-16-0234.1.

—_, and J. M. Peters, 2018: Theoretical expressions for the ascent rate of moist convective thermals. J. Atmos. Sci., 75, 1699-1719, https://doi.org/10.1175/JAS-D-17-0295.1.

—, J. A. Curry, and V. I. Khvorostyanov, 2005: A new doublemoment microphysics parameterization for application in cloud and climate models. Part I: Description. J. Atmos. Sci., 62, 1665-1677, https://doi.org/10.1175/JAS3446.1.

— J. M. Peters, W. M. Hannah, A. C. Varble, and S. E. Giangrande, 2020: Thermal chains and entrainment in cumulus updrafts. Part I: Theoretical description. J. Atmos. Sci., 77, 3637-3660, https://doi.org/10.1175/JAS-D-19-0243.1.

Morton, B. R., G. I. Taylor, and J. S. Turner, 1956: Turbulent gravitational convection from maintained and instantaneous sources. Proc. Roy. Soc. London, 234A, 1-23, https://doi.org/ 10.1098/rspa.1956.0011.

Moser, D. H., and S. Lasher-Trapp, 2017: The influence of successive thermals on entrainment and dilution in a simulated cumulus congestus. J. Atmos. Sci., 74, 375-392, https://doi.org/ 10.1175/JAS-D-16-0144.1.

Peters, J. M., W. M. Hannah, and H. Morrison, 2019: The influence of vertical wind shear on moist thermals. J. Atmos. Sci., 76, 1645-1659, https://doi.org/10.1175/JAS-D-18-0296.1.

Raymond, D. J., and A. M. Blyth, 1989: Precipitation development in a New Mexico thunderstorm. Quart. J. Roy. Meteor. Soc., 115, 1397-1423, https://doi.org/10.1002/qj.49711549011.

Romps, D. M., 2010: A direct measure of entrainment. J. Atmos. Sci., 67, 1908-1927, https://doi.org/10.1175/2010JAS3371.1.
— , and A. B. Charn, 2015: Sticky thermals: Evidence for a dominant balance between buoyancy and drag in cloud updrafts. J. Atmos. Sci., 72, 2890-2901, https://doi.org/10.1175/ JAS-D-15-0042.1.

- , and R. Oktem, 2015: Stereo photogrammetry reveals substantial drag on cloud thermals. Geophys. Res. Lett., 42, 5051-5057, https://doi.org/10.1002/2015GL064009.

Scorer, R. S., 1957: Experiments on convection of isolated masses of buoyant fluid. J. Fluid Mech., 2, 583-594, https://doi.org/ 10.1017/S0022112057000397.

— vection. Quart. J. Roy. Meteor. Soc., 79, 94-103, https://doi.org/ 10.1002/qj.49707933908.

Sherwood, S. C., D. Hernandez-Deckers, and M. Colin, 2013: Slippery thermals and the cumulus entrainment paradox. J. Atmos. Sci., 70, 2426-2442, https://doi.org/10.1175/JAS-D12-0220.1.

Squires, P., and J. S. Turner, 1962: An entraining jet model for cumulo-nimbus updrafts. Tellus, 14A, 422-434, https://doi.org/ 10.1111/j.2153-3490.1962.tb01355.x.

Turner, J. S., 1962: The 'starting plume' in neutral surroundings. J. Fluid Mech., 13, 356-368, https://doi.org/10.1017/S0022112062000762.

Weisman, M. L., and J. B. Klemp, 1982: The dependence of numerically simulated convective storms on vertical wind shear and buoyancy. Mon. Wea. Rev., 110, 504-520, https://doi.org/ 10.1175/1520-0493(1982)110<0504:TDONSC >2.0.CO;2.

Yano, J. I., 2014: Basic convective element: Bubble or plume? A historical review. Atmos. Chem. Phys., 14, 7019-7030, https:// doi.org/10.5194/acp-14-7019-2014.

Zhou, L., B. Neale, M. Jochum, and R. Murtugudde, 2012: Improved Madden-Julian oscillations with improved physics: The impact of modified convective parameterizations. J. Climate, 25, 11161136, https://doi.org/10.1175/2011JCLI4059.1. 